UWO-TH-08/13

DAMTP-2008-67

\title{
Second order hydrodynamics of a CFT plasma from boost invariant expansion
}

\author{
Alex Buchel ${ }^{1,2}$ and Miguel Paulos 3 \\ ${ }^{1}$ Department of Applied Mathematics \\ University of Western Ontario \\ London, Ontario N6A 5B\%, Canada \\ ${ }^{2}$ Perimeter Institute for Theoretical Physics \\ Waterloo, Ontario N2J 2W9, Canada \\ ${ }^{3}$ Department of Applied Mathematics and \\ Theoretical Physics, Cambridge CB3 0WA, U.K.
}

\begin{abstract}
We compute finite coupling correction to a nonlinear second order hydrodynamic coefficient in the boost invariant expansion of the $\mathcal{N}=4$ supersymmetric Yang-Mills plasma. The result is universal for a large class of strongly coupled four dimensional conformal gauge theories.
\end{abstract}

August 2008 


\section{Introduction and summary}

It is common to model the boost-invariant expansion [1] of the strongly coupled (relativistic) quark-gluon plasma (sQGP) produced in heavy ion collisions at RHIC in the framework of Mueller-Israel-Stewart theory (MIS) [2, 3]. One often further approximates sQGP dynamics in the relevant regime as that of a conformal theory. For a four-dimensional viscous conformal plasma undergoing boost-invariant expansion, MIS theory predicts the evolution of the energy density $\epsilon(\tau)$ and the component of the viscous flow $\Phi(\tau)$ as (see [4])

$$
\begin{aligned}
\partial_{\tau} \epsilon & =-\frac{4 \epsilon}{3 \tau}+\frac{\Phi}{\tau} \\
\tau_{\Pi} \partial_{\tau} \Phi & =\frac{4 \eta}{3 \tau}-\Phi-\frac{4 \tau_{\Pi}}{3 \tau} \Phi
\end{aligned}
$$

where the two phenomenological parameters are: $\eta \propto T^{3}$, the plasma shear viscosity, and $\tau_{\Pi} \propto T^{-1}$, the plasma relaxation time.

To a large extent motivated and guided by the gauge theory/string theory correspondence of Maldacena [5, 6, 7], Baier et.al [8] and Bhattacharyya et.al [9] recently formulated a complete theory of the second order relativistic viscous hydrodynamics of conformal fluids. In this theory, the MIS linearized equations governing the boostinvariant expansion (1.1) are modified by the inclusion of a new term, quadratic in the component $\Phi$ of the viscous flow:

$$
\begin{aligned}
\partial_{\tau} \epsilon & =-\frac{4 \epsilon}{3 \tau}+\frac{\Phi}{\tau} \\
\tau_{\Pi} \partial_{\tau} \Phi & =\frac{4 \eta}{3 \tau}-\Phi-\frac{4 \tau_{\Pi}}{3 \tau} \Phi-\frac{\lambda_{1}}{2 \eta^{2}} \Phi^{2}
\end{aligned}
$$

where $\lambda_{1} \propto T^{2}$ is a new second order hydrodynamic coefficient. An important observation of $[8,9]$ was that for the $\mathcal{N}=4$ supersymmetric Yang-Mills plasma (SYM), and in fact for all (infinitely) strongly coupled conformal gauge theory plasmas, allowing for a dual string theory description, $\lambda_{1} \neq 0$. Moreover, for the near-equilibrium dynamics ${ }^{1}$ (where one can reasonably apply hydrodynamics at all) the nonlinear term in (1.2) is equally important to $\tau_{\Pi}$ terms of the MIS theory, introduced to restore causality in first-order hydrodynamics. As reported in [8, 9], and for the shear viscosity in [11], any (infinitely) strongly coupled four-dimensional conformal gauge theory plasma (with a

\footnotetext{
${ }^{1}$ The near-equilibrium dynamics corresponds to the late-time boost-invariant expansion (see [10]).
} 
string theory dual) has

$$
\frac{\eta}{s}=\frac{1}{4 \pi}, \quad \tau_{\Pi} T=\frac{2-\ln 2}{2 \pi}, \quad \frac{\lambda_{1} T}{\eta}=\frac{1}{2 \pi},
$$

where $s$ is the entropy density.

The finite coupling corrections to $\eta / s$ and $\tau_{\Pi} T$ for $\mathcal{N}=4$ SYM plasma were computed in $[12,13,14,15,16]$ and [18]:

$$
\frac{\eta}{s}=\frac{1}{4 \pi}\left(1+\frac{120}{8} \zeta(3) \lambda^{-3 / 2}+\cdots\right), \quad \tau_{\Pi} T=\frac{2-\ln 2}{2 \pi}+\frac{375}{32 \pi} \zeta(3) \lambda^{-3 / 2}+\cdots,
$$

where $\lambda$ is the gauge theory 't Hooft coupling. It was proposed in $[19]^{2}$ that corrections (1.4) are universal for all four dimensional conformal gauge theory plasmas (with equal $a$ and $c$ central charges) that allow for string theory duals. Similarly, within the same class of conformal plasmas, the finite coupling correction to $\lambda_{1}$ is universal.

In this paper we report the (universal) finite coupling correction to $\lambda_{1}$. We find

$$
\frac{\lambda_{1} T}{\eta}=\frac{1}{2 \pi}\left(1+\frac{215}{8} \zeta(3) \lambda^{-3 / 2}+\cdots\right)
$$

Given (1.4) and (1.5) we have now a complete set of universal phenomenological parameters describing boost-invariant expansion of conformal gauge theory plasmas at finite 't Hooft coupling. We hope these results will prove useful in numerical hydrodynamic simulations of RHIC (and LHC) nuclear collisions.

The computations are quite technical, so we present only relevant steps and for the details refer the reader to previous work on the subject. We rely on the pioneering work of Janik and Peschanski [21] (and important further developments in $[22,23,24]$ ) which sets up a to study boost invariant expansion of a gauge theory plasma in a dual string theory setting. We use notations and results (often without quoting them here due to their technical nature) of [15]. Some further technical details appear in Appendices A and B, and supplemental data is available as [25].

We would like to comment on the issue of singularities in the Janik-Peschanski framework. To extract $\lambda_{1}$ we need to go to the third order in the late proper time expansion of the dual string theory background $[24,8]$. This is the first time where the singularities of the dual background geometry can not be completely removed by

\footnotetext{
${ }^{2}$ The proof is given in [20].
} 
appropriately adjusting the hydrodynamic parameters [24, 26]. Specifically, appropriate hydrodynamic parameters will remove all the pole singularities in the curvature invariants in the late time expansion, but the logarithmic singularities will persist in quadratic (and higher order) Riemann tensor invariants. It was understood in [27] (also independently in [28]) that a singular proper time redefinition in the bulk will remove all the singularities identified in $[24,26]$. Since such proper time redefinition has only a logarithmic singularity, it can not affect the condition for the absence of pole singularities which determines the hydrodynamic parameters. Thus, we are justified to use the original framework of Janik-Peschanski to extract $\lambda_{1}$.

Given the complexity of the computations, we feel that an independent check on the analysis is important. Thus, while computations in Secs. 2-4 are done in the Janik-Peschanski framework, in Sec. 5 we reanalyze boost-invariant expansion of a conformal gauge theory plasma in singularity-free approach of Kinoshita et al [28]. Both approaches lead to the same value of $\lambda_{1}$.

\section{Janik-Peschanski dual to a boost invariant plasma expan- sion}

The framework to study string theory duals of boost-invariant plasmas was proposed in [21]. Here we closely follow notations and analysis in [26] and [15]. Most details ${ }^{3}$ (including the description of the computational framework) are omitted due to their technical nature and the fact that they have already been explained in [15].

The string theory background holographically dual to a Bjorken flow of the $\mathcal{N}=4$ plasma takes the form $[21,26,15]$

$$
\begin{aligned}
d \tilde{s}_{10}^{2} & =\tilde{g}_{M N} d \xi^{M} d \xi^{N}= \\
& =e^{-2 \alpha(\tau, z)} g_{\mu \nu}(x) d x^{\mu} d x^{\nu}+e^{6 / 5 \alpha(\tau, z)}\left(d S^{5}\right)^{2}
\end{aligned}
$$

where $\left(d S^{5}\right)^{2}$ is the line element for a 5 -dimensional sphere with unit radius, and

$$
\begin{aligned}
d s^{2} & =g_{\mu \nu} d x^{\mu} d x^{\nu}= \\
& =\frac{1}{z^{2}}\left[-e^{2 a(\tau, z)} d \tau^{2}+e^{2 b(\tau, z)} \tau^{2} d y^{2}+e^{2 c(\tau, z)} d x_{\perp}^{2}\right]+\frac{d z^{2}}{z^{2}}
\end{aligned}
$$

\footnotetext{
${ }^{3}$ The details of the analysis are available from the authors upon request.
} 
where $d x_{\perp}^{2} \equiv d x_{1}^{2}+d x_{2}^{2}$. The 5 -form $F_{5}$ takes form ${ }^{4}$

$$
F_{5}=\mathcal{F}_{5}+\star \mathcal{F}_{5}, \quad \mathcal{F}_{5}=-4 \omega_{S^{5}}
$$

where $\omega_{S^{5}}$ is the 5-sphere volume form. Moreover, the dilaton is $\phi=\phi(\tau, z)$.

Equations of motion for the metric warp factors and the dilaton are solved as a series expansion in the late proper time $\tau \rightarrow \infty$, but exactly in the scaling variable [21]

$$
v \equiv \frac{z}{\tau^{1 / 3}}
$$

Specifically,

$$
\begin{aligned}
a(\tau, v)= & \left(a_{0}(v)+\gamma \hat{a}_{0}(v)\right)+\frac{1}{\tau^{2 / 3}}\left(a_{1}(v)+\gamma \hat{a}_{1}(v)\right)+\frac{1}{\tau^{4 / 3}}\left(a_{2}(v)+\gamma \hat{a}_{2}(v)\right) \\
& +\frac{1}{\tau^{2}}\left(a_{3}(v)+\gamma \hat{a}_{3}(v)\right), \\
b(\tau, v)= & \left(b_{0}(v)+\gamma \hat{b}_{0}(v)\right)+\frac{1}{\tau^{2 / 3}}\left(b_{1}(v)+\gamma \hat{b}_{1}(v)\right)+\frac{1}{\tau^{4 / 3}}\left(b_{2}(v)+\gamma \hat{b}_{2}(v)\right) \\
& +\frac{1}{\tau^{2}}\left(b_{3}(v)+\gamma \hat{b}_{3}(v)\right), \\
c(\tau, v)= & \left(c_{0}(v)+\gamma \hat{c}_{0}(v)\right)+\frac{1}{\tau^{2 / 3}}\left(c_{1}(v)+\gamma \hat{c}_{1}(v)\right)+\frac{1}{\tau^{4 / 3}}\left(c_{2}(v)+\gamma \hat{c}_{2}(v)\right) \\
& +\frac{1}{\tau^{2}}\left(c_{3}(v)+\gamma \hat{c}_{3}(v)\right), \\
\alpha(\tau, v)= & \gamma \hat{\alpha}_{0}(v)+\frac{1}{\tau^{2 / 3}} \gamma \hat{\alpha}_{1}(v)+\frac{1}{\tau^{4 / 3}} \gamma \hat{\alpha}_{2}(v)+\frac{1}{\tau^{2}} \gamma \hat{\alpha}_{3}(v), \\
\phi(\tau, v)= & \gamma \hat{\phi}_{0}(v)+\frac{1}{\tau^{2 / 3}} \gamma \hat{\phi}_{1}(v)+\frac{1}{\tau^{4 / 3}} \gamma \hat{\phi}_{2}(v)+\frac{1}{\tau^{2}} \gamma \hat{\phi}_{3}(v),
\end{aligned}
$$

where

$$
\gamma=\frac{1}{8} \zeta(3)\left(\alpha^{\prime}\right)^{3} \quad \Leftrightarrow \quad \frac{1}{8} \zeta(3) \lambda^{-3 / 2}
$$

is the leading string theory $\alpha^{\prime}$-correction to type IIB supergravity. As argued in $[16],[17]$, the five-form is not corrected to this order.

Solutions for the background warp factors $\left\{a_{i}, b_{i}, c_{i}\right\}, i=0,1,2,3$ were obtained in [24]; the leading string theory $\alpha^{\prime}$-corrections, up to the second order, $\left\{\hat{a}_{i}, \hat{b}_{i}, \hat{c}_{i}, \hat{\alpha}_{i}, \hat{\phi}_{i}\right\}$ with $i=0,1,2$, were discussed in [15]. In the next section, we extended the analysis of [15] to the leading string theory $\alpha^{\prime}$-corrections to the supergravity background at the third order, i.e., for $\hat{a}_{3}, \hat{b}_{3}, \hat{c}_{3}, \hat{\alpha}_{3}, \hat{\phi}_{3}$.

\footnotetext{
${ }^{4}$ We normalize the five-form flux so that the asymptotic AdS radius is one.
} 


\section{Equations of motion and solutions for $\left\{\hat{a}_{3}, \hat{b}_{3}, \hat{c}_{3}, \hat{\alpha}_{3}, \hat{\phi}_{3}\right\}$}

We obtain equations of motion (including the constraints) at the third order for $\hat{a}_{3}, \hat{b}_{3}$, $\hat{c}_{3}, \hat{\alpha}_{3}, \hat{\phi}_{3}$, extending the analysis in [15]. All the equations must be solved with the boundary conditions

$$
\left.\left\{\hat{a}_{3}(v), \hat{b}_{3}(v), \hat{c}_{3}(v), \hat{\alpha}_{3}(v), \hat{\phi}_{3}(v)\right\}\right|_{v \rightarrow 0}=0 .
$$

We find the following set of equations for the next-to-next-to-next-to-leading order in the late proper time expansion at order $\mathcal{O}(\gamma)$

$$
\begin{gathered}
0=\hat{c}_{3}^{\prime \prime}+\frac{1}{2} \hat{b}_{3}^{\prime \prime}+\frac{5 v^{4}-9}{\left(3+v^{4}\right) v} \hat{c}_{3}^{\prime}+\frac{5 v^{4}-9}{2\left(3+v^{4}\right) v} \hat{b}_{3}^{\prime}+\mathcal{S}_{(3,1)} \\
0=\hat{c}_{3}^{\prime \prime}+\frac{1}{2} \hat{a}_{3}^{\prime \prime}+\frac{5 v^{8}+27}{v\left(v^{8}-9\right)} \hat{c}_{3}^{\prime}+\frac{9+5 v^{4}}{2 v\left(v^{4}-3\right)} \hat{a}_{3}^{\prime}+\mathcal{S}_{(3,2)} \\
0=\hat{c}_{3}^{\prime \prime}+\hat{a}_{3}^{\prime \prime}+\hat{b}_{3}^{\prime \prime}+\frac{9+5 v^{4}}{v\left(v^{4}-3\right)} \hat{a}_{3}^{\prime}+\frac{5 v^{8}+27}{v\left(v^{8}-9\right)} \hat{b}_{3}^{\prime}+\frac{5 v^{8}+27}{v\left(v^{8}-9\right)} \hat{c}_{3}^{\prime}+\mathcal{S}_{(3,3)}, \\
0=\hat{c}_{3}^{\prime \prime}+\frac{1}{2} \hat{b}_{3}^{\prime \prime}-\frac{3\left(v^{4}-3\right)}{2\left(3+v^{4}\right) v} \hat{a}_{3}^{\prime}+\frac{3\left(v^{8}-5 v^{4}-6\right)}{v\left(v^{8}-9\right)} \hat{b}_{3}^{\prime}+\frac{3\left(3 v^{8}-10 v^{4}-21\right)}{v\left(v^{8}-9\right)} \hat{c}_{3}^{\prime}-\frac{72 v^{2}}{v^{8}-9} \hat{b}_{3} \\
-\frac{144 v^{2}}{v^{8}-9} \hat{c}_{3}+\mathcal{S}_{(3,4)}, \\
0=\hat{c}_{3}^{\prime}+\frac{1}{2} \hat{b}_{3}^{\prime}+\frac{\left(v^{4}-3\right)^{2}}{2\left(v^{4}-2 v^{2}+3\right)\left(v^{4}+2 v^{2}+3\right)} \hat{a}_{3}^{\prime}+\mathcal{S}_{(3,5)} \\
0=\hat{\alpha}_{3}^{\prime \prime}+\frac{5 v^{8}+27}{v\left(v^{8}-9\right)} \hat{\alpha}_{3}^{\prime}-\frac{32}{v^{2}} \hat{\alpha}_{3}+\mathcal{S}_{(3,6)} \\
0=\hat{\phi}_{3}^{\prime \prime}+\frac{5 v^{8}+27}{v\left(v^{8}-9\right)} \hat{\phi}_{3}^{\prime}+\mathcal{S}_{(3,7)}
\end{gathered}
$$

where the source terms $\left\{\mathcal{S}_{(3,1)} \cdots, \mathcal{S}_{(3,7)}\right\}$ are given in [25]. While the system (3.2)-(3.8) is overdetermined, we explicitly verified that it is consistent.

Solving (3.2)-(3.8) is quite complicated. Fortunately, we do not need a complete solution. Our ultimate goal is to determine $C$ from the nonsingularity of the ten dimensional metric curvature invariants to order $\mathcal{O}(\gamma)$ and to order $\mathcal{O}\left(\tau^{-2}\right)$ in the late proper time expansion. Thus we evaluate metric invariants first, find what field combinations affect the singularity as $v \rightarrow 3_{-}^{1 / 4}$, and then solve just for those combinations of fields. 
We assume that

$$
\hat{\eta}=\hat{\eta}_{0}+\gamma \hat{\eta}_{1}+\mathcal{O}\left(\gamma^{2}\right), \quad C=C_{0}+\gamma C_{1}+\mathcal{O}\left(\gamma^{2}\right)
$$

and evaluate background curvature invariants to order $\mathcal{O}(\gamma)$ near

$$
x \equiv 3^{1 / 4}-v .
$$

We use explicit solutions at lower orders, as well as equations of motion for the second and the third order to eliminate the derivatives (if possible) of

$$
\left\{a_{3}, b_{3}, c_{3} ; \hat{a}_{2}, \hat{b}_{2}, \hat{c}_{2}, \hat{\alpha}_{2} ; \hat{a}_{3}, \hat{b}_{3}, \hat{c}_{3}, \hat{\alpha}_{3}\right\}
$$

from the curvature invariants (see [15] for details).

\section{$3.1 \mathcal{R}$ at order $\mathcal{O}\left(\tau^{-2}\right)$}

For the Ricci scalar we find

$$
\begin{aligned}
\mathcal{R}= & \cdots+\frac{\gamma}{\tau^{2}\left(v^{4}+2 v^{2}+3\right)\left(v^{4}-2 v^{2}+3\right)\left(3+v^{4}\right)^{3}}\left\{-\frac{207360 v^{13}\left(v^{4}-3\right)^{3}}{\left(3+v^{4}\right)^{4}} a_{3}^{\prime}\right. \\
& -\frac{497664 \hat{\eta}_{0} v^{13}}{\left(v^{4}+2 v^{2}+3\right)\left(v^{4}-2 v^{2}+3\right)\left(v^{8}-9\right)\left(3+v^{4}\right)^{4}}\left(2 v^{28}-56 v^{24}+89 v^{20}-765 v^{16}\right. \\
& \left.+900 v^{12}-9882 v^{8}+7857 v^{4}-6561\right) a_{2}^{\prime}-\frac{13824 v^{14} \hat{\eta}_{0}}{\left(v^{8}-9\right)^{4}\left(3+v^{4}\right)}\left(25 v^{28}+324 \hat{\eta}_{0}^{2} v^{26}\right. \\
& +189 v^{24}+6696 v^{22} \hat{\eta}_{0}^{2}+1269 v^{20}+69660 \hat{\eta}_{0}^{2} v^{18}+7641 v^{16}-58320 v^{14} \hat{\eta}_{0}^{2}+22923 v^{12} \\
& +626940 \hat{\eta}_{0}^{2} v^{10}+34263 v^{8}+542376 \hat{\eta}_{0}^{2} v^{6}+45927 v^{4}+236196 \hat{\eta}_{0}^{2} v^{2} \\
& +54675) \ln \frac{3-v^{4}}{3+v^{4}}+\frac{1990656 \hat{\eta}_{0} v^{16}\left(b_{2}+2 c_{2}\right)}{\left(v^{8}-9\right)^{4}\left(3+v^{4}\right)}\left(3 v^{24}+62 v^{20}+645 v^{16}-540 v^{12}\right. \\
& \left.+5805 v^{8}+5022 v^{4}+2187\right)-\frac{1492992 v^{16} \hat{\eta}_{0}^{3}}{\left(v^{4}-2 v^{2}+3\right)\left(v^{4}+2 v^{2}+3\right)\left(3+v^{4}\right)^{3}\left(v^{8}-9\right)^{5}} \\
& \times\left(37 v^{48}+4434912 v^{24}+14348907+1273320 v^{20}+7452 v^{36}+315801 v^{32}\right. \\
& +46008 v^{28}+17684 v^{40}-36137988 v^{4}-300 v^{44}+12538071 v^{16}-28072332 v^{12} \\
& \left.+78180876 v^{8}\right)+\frac{27648 \hat{\eta}_{0} v^{18}}{\left(v^{4}-2 v^{2}+3\right)\left(v^{4}+2 v^{2}+3\right)\left(v^{8}-9\right)^{5}}\left(53 v^{36}-533 v^{32}\right. \\
& +6978 v^{28}+4518 v^{24}+123228 v^{20}+112428 v^{16}+1289358 v^{12}+1255338 v^{8} \\
& \left.\left.+693279 v^{4}+4153113\right)\right\},
\end{aligned}
$$


where $\cdots$ denote lower orders in the later time expansion. We recall explicit expressions for $\left\{a_{2}, b_{2}, c_{2}\right\}$ as well as present the decoupled equation for $a_{3}$ in Appendix A. Using (A.1), (A.4) we find

$$
\begin{aligned}
\mathcal{R}= & \cdots+\frac{\gamma}{\tau^{2}}\left\{-3 \hat{\eta}_{0} 3^{1 / 4}\left(\sqrt{3}-18 \hat{\eta}_{0}^{2}\right) \frac{1}{x^{5}}+\frac{3}{4} \hat{\eta}_{0}\left(13 \sqrt{3}-4 \ln 2 \sqrt{3}-30 \hat{\eta}_{0}^{2}+2 C_{0}\right) \frac{1}{x^{4}}\right. \\
& -\frac{1}{4} \hat{\eta}_{0} 3^{1 / 4}\left(426 \hat{\eta}_{0}^{2} \sqrt{3}+4 C_{0} \sqrt{3}-3-24 \ln 2\right) \frac{1}{x^{3}} \\
& -\frac{1}{4} \hat{\eta}_{0}\left(75-78 \ln 2+876 \hat{\eta}_{0}^{2} \sqrt{3}+13 C_{0} \sqrt{3}\right) \frac{1}{x^{2}} \\
& \left.+\frac{15}{4} \hat{\eta}_{0} 3^{1 / 4}\left(\sqrt{3}-2 \ln 2 \sqrt{3}+84 \hat{\eta}_{0}^{2}+C_{0}\right) \frac{1}{x}-64 \sqrt{3} \hat{\eta}_{0} \ln x+\text { finite }\right\} .
\end{aligned}
$$

From (3.12) we find that the Ricci scalar of the string theory geometry does not have pole singularities as $x \rightarrow 0_{+}$when

$$
\hat{\eta}_{0}=\frac{1}{2^{1 / 2} 3^{3 / 4}}, \quad C_{0}=2 \sqrt{3} \ln 2-\frac{17}{\sqrt{3}},
$$

which are precisely the conditions found from the nonsingularity of the Riemann tensor squared [23, 24], as well as higher curvature invariants [26], in the supergravity approximation to the string theory dual of the $\mathcal{N}=4$ SYM Bjorken flow. The difference here (compare to $[23,24,26])$ is that $\hat{\eta}_{0}$ and $C_{0}$ are already fixed by requiring the nonsingularity of the Ricci scalar.

While the pole singularities in the ten dimensional Ricci scalar are removed, given (3.13), the logarithmic singularity still persists. A similar observation was made at the supergravity level for the Riemann tensor invariants in $[24,26]$. We expect that the remaining logarithmic singularity is removed by an appropriate change of variable $[27,28]$. 


\section{$3.2 \mathcal{R}_{\mu \nu \rho \lambda} \mathcal{R}^{\mu \nu \rho \lambda}$ at order $\mathcal{O}\left(\tau^{-2}\right)$}

A bit more work is necessary to determine the nonsingularity condition of the Riemann tensor squared at order $\mathcal{O}(\gamma)$. Generalizing the notation of [26]

$$
\begin{aligned}
\mathcal{I}^{[2]} & \equiv \mathcal{R}_{\mu \nu \rho \lambda} \mathcal{R}^{\mu \nu \rho \lambda} \\
& =\left(\mathcal{I}_{0}^{[2] S U G R A}(v)+\gamma \mathcal{I}_{0}^{[2] W}(v)\right)+\frac{1}{\tau^{2 / 3}}\left(\mathcal{I}_{1}^{[2] S U G R A}(v)+\gamma \mathcal{I}_{1}^{[2] W}(v)\right) \\
& +\frac{1}{\tau^{4 / 3}}\left(\mathcal{I}_{2}^{[2] S U G R A}(v)+\gamma \mathcal{I}_{2}^{[2] W}(v)\right)+\frac{1}{\tau^{2}}\left(\mathcal{I}_{3}^{[2] S U G R A}(v)+\gamma \mathcal{I}_{3}^{[2] W}(v)\right) \\
& +\mathcal{O}\left(\tau^{-8 / 3}\right)+\mathcal{O}\left(\gamma^{2}\right) .
\end{aligned}
$$

Explicitly we find:

$$
\begin{aligned}
& \mathcal{I}_{3}^{[2] S U G R A}=\frac{1}{\left(v^{4}+2 v^{2}+3\right)\left(v^{4}-2 v^{2}+3\right)}\left\{\frac{1152 v^{5}\left(v^{4}-3\right)^{3}}{\left(3+v^{4}\right)^{3}} a_{3}^{\prime}\right. \\
& -\frac{13824 v^{5} \hat{\eta}}{\left(3+v^{4}\right)^{3}\left(v^{8}-9\right)\left(v^{4}+2 v^{2}+3\right)\left(v^{4}-2 v^{2}+3\right)}\left(8 v^{24}+17 v^{20}+133 v^{16}+210 v^{12}\right. \\
& \left.+1674 v^{8}-243 v^{4}+729\right) a_{2}^{\prime}-\frac{55296 \hat{\eta} v^{8}}{\left(v^{8}-9\right)^{4}}\left(v^{24}+18 v^{20}+183 v^{16}+60 v^{12}+1647 v^{8}\right. \\
& \left.+1458 v^{4}+729\right)\left(b_{2}+2 c_{2}\right)+\frac{384 v^{6} \hat{\eta}}{\left(v^{8}-9\right)^{4}}\left(5 v^{28}+108 \hat{\eta}^{2} v^{26}+57 v^{24}+1944 v^{22} \hat{\eta}^{2}\right. \\
& +465 v^{20}+19764 \hat{\eta}^{2} v^{18}+2565 v^{16}+6480 v^{14} \hat{\eta}^{2}+7695 v^{12}+177876 \hat{\eta}^{2} v^{10}+12555 v^{8} \\
& \left.+157464 \hat{\eta}^{2} v^{6}+13851 v^{4}+78732 \hat{\eta}^{2} v^{2}+10935\right) \ln \frac{3-v^{4}}{3+v^{4}} \\
& +\frac{82944 v^{12} \hat{\eta}^{3}}{\left(v^{8}-9\right)^{5}\left(v^{4}-2 v^{2}+3\right)\left(v^{4}+2 v^{2}+3\right)\left(3+v^{4}\right)^{2}}\left(434268 v^{20}+794286 v^{16}+514674 v^{12}\right. \\
& +2322594 v^{4}-334611 v^{8}-531441+114642 v^{24}+37740 v^{28}+49 v^{40}+2 v^{44}+1314 v^{36} \\
& \left.+7251 v^{32}\right)-\frac{768 \hat{\eta} v^{10}}{\left(v^{4}-3\right)\left(v^{4}-2 v^{2}+3\right)\left(v^{4}+2 v^{2}+3\right)\left(v^{8}-9\right)^{4}}\left(v^{36}-25 v^{32}+1026 v^{28}\right. \\
& \left.\left.+6054 v^{24}+33300 v^{20}+88452 v^{16}+338094 v^{12}+392202 v^{8}+317115 v^{4}+452709\right)\right\}
\end{aligned}
$$




$$
\begin{aligned}
& \mathcal{I}_{3}^{[2] W}=\frac{1152 v^{5}\left(v^{4}-3\right)^{3}}{\left(v^{4}+2 v^{2}+3\right)\left(v^{4}-2 v^{2}+3\right)\left(3+v^{4}\right)^{3}} \hat{a}_{3}^{\prime}-\frac{192}{\left(3+v^{4}\right)^{4}}\left(5 v^{16}+60 v^{12}+54 v^{8}\right. \\
& \left.+540 v^{4}+405\right) \hat{\alpha}_{3}-\frac{13824 v^{5} \hat{\eta}_{0}}{\left(v^{4}+2 v^{2}+3\right)^{2}\left(v^{4}-2 v^{2}+3\right)^{2}\left(v^{4}+3\right)^{3}\left(v^{8}-9\right)}\left(8 v^{24}\right. \\
& \left.+17 v^{20}+133 v^{16}+210 v^{12}+1674 v^{8}-243 v^{4}+729\right) \hat{a}_{2}^{\prime} \\
& -\frac{55296 \hat{\eta}_{0} v^{8}}{\left(v^{8}-9\right)^{4}\left(v^{4}+2 v^{2}+3\right)\left(v^{4}-2 v^{2}+3\right)}\left(v^{24}+18 v^{20}+183 v^{16}+60 v^{12}\right. \\
& \left.+1647 v^{8}+1458 v^{4}+729\right)\left(\hat{b}_{2}+2 \hat{c}_{2}\right)+\frac{165888 \hat{\eta}_{0} v^{8}\left(v^{4}-3\right)}{\left(3+v^{4}\right)^{5}} \hat{\alpha}_{2} \\
& -\frac{192 v^{5} \mathcal{Q}_{1}}{\left(3+v^{4}\right)^{9}\left(v^{4}+2 v^{2}+3\right)^{2}\left(v^{4}-2 v^{2}+3\right)^{2}} a_{3}^{\prime} \\
& +\frac{768 v^{5} \mathcal{Q}_{2}}{\left(3+v^{4}\right)^{7}\left(v^{8}-9\right)^{3}\left(v^{4}+2 v^{2}+3\right)^{3}\left(v^{4}-2 v^{2}+3\right)^{3}} a_{2}^{\prime} \\
& +\frac{3072 \mathcal{Q}_{3} v^{8}}{\left(3+v^{4}\right)^{4}\left(v^{8}-9\right)^{6}\left(v^{4}+2 v^{2}+3\right)^{2}\left(v^{4}-2 v^{2}+3\right)^{2}}\left(b_{2}+2 c_{2}\right) \\
& +\frac{64 \mathcal{Q}_{4} v^{6}}{3\left(3+v^{4}\right)^{4}\left(v^{8}-9\right)^{6}\left(v^{4}+2 v^{2}+3\right)^{2}\left(v^{4}-2 v^{2}+3\right)^{2}} \ln \frac{3-v^{4}}{3+v^{4}} \\
& +\frac{128 \mathcal{Q}_{5} v^{10} \hat{\eta}_{0} \delta_{1}}{\left(3+v^{4}\right)^{2}\left(v^{8}-9\right)^{6}\left(v^{4}+2 v^{2}+3\right)^{3}\left(v^{4}-2 v^{2}+3\right)^{3}} \\
& -\frac{128 \mathcal{Q}_{6} v^{10} \delta_{2}}{3\left(v^{8}-9\right)^{5}\left(v^{4}-2 v^{2}+3\right)^{2}\left(v^{4}+2 v^{2}+3\right)^{2}\left(3+v^{4}\right)^{2}} \\
& -\frac{1492992 v^{12} \mathcal{Q}_{7} \hat{\eta}_{0}^{3}}{\left(v^{4}-2 v^{2}+3\right)^{3}\left(v^{4}+2 v^{2}+3\right)^{3}\left(3+v^{4}\right)^{5}\left(v^{8}-9\right)^{8}} \\
& +\frac{4608 \hat{\eta}_{0} v^{10} \mathcal{Q}_{8}}{\left(v^{4}-2 v^{2}+3\right)^{3}\left(v^{4}+2 v^{2}+3\right)^{3}\left(3+v^{4}\right)^{2}\left(v^{8}-9\right)^{8}},
\end{aligned}
$$

where $\left\{\mathcal{Q}_{1}, \cdots \mathcal{Q}_{8}\right\}$ are given in Appendix B. Appendix B also contains explicit expressions for $\hat{a}_{2}^{\prime}, f_{2} \equiv \hat{c}_{2}+\frac{1}{2} \hat{b}_{2}$ [15], and the equation of motion for $\hat{a}_{3}$. As in the case of $\hat{\alpha}_{2}$, although we can not explicitly solve for $\hat{\alpha}_{3}$, we can argue that $\hat{\alpha}_{3}(v)$ can be chosen to be finite (along with its first derivative) as $v \rightarrow 3_{-}^{1 / 4}$, while having a vanishing nonnormalizable mode as $v \rightarrow 0_{+}$.

We now have all the necessary ingredients to determine $\hat{\eta}_{1}, C_{1}$ from the nonsingularity of $\left(\mathcal{I}_{3}^{[2] S U G R A}(v)+\gamma \mathcal{I}_{3}^{[2] W}(v)\right)$ : using results of Appendices $\mathrm{A}$ and $\mathrm{B}$, as well as 
(3.13), we find

$$
\begin{aligned}
& \mathcal{I}_{3}^{[2] S U G R A}+\left.\gamma \mathcal{I}_{3}^{[2] W}\right|_{x \equiv 3^{1 / 4}-v \rightarrow 0_{+}}=\frac{\sqrt{2}}{18} \gamma\left(7182+4 \sqrt{2} 3^{3 / 4} \delta_{2}-15 \delta_{1}-72 \sqrt{2} 3^{3 / 4} \hat{\eta}_{1}\right) \frac{1}{x^{5}} \\
& -\frac{1}{24} \gamma\left(4 \sqrt{2} 3^{3 / 4} \delta_{1} \ln 2-1272 \sqrt{2} 3^{3 / 4} \ln 2+30090 \sqrt{2} 3^{3 / 4}+24 \sqrt{3} \delta_{2}-21 \sqrt{2} 3^{3 / 4} \delta_{1}\right. \\
& \left.+83^{1 / 4} \sqrt{2} C_{1}-176 \sqrt{3} \hat{\eta}_{1}+32 \sqrt{2} 3^{1 / 4} \delta_{3}\right) \frac{1}{x^{4}}+\frac{3^{1 / 4}}{72} \gamma\left(24 \delta_{2}+1104 \hat{\eta}_{1}+16 \sqrt{2} 3^{3 / 4} C_{1}\right. \\
& \left.-51 \sqrt{2} 3^{1 / 4} \delta_{1}-7632 \sqrt{2} 3^{1 / 4} \ln 2+144630 \sqrt{2} 3^{1 / 4}+64 \sqrt{2} 3^{3 / 4} \delta_{3}+24 \sqrt{2} 3^{1 / 4} \delta_{1} \ln 2\right) \frac{1}{x^{3}} \\
& +\frac{1}{36} \gamma\left(-4 \delta_{2}+264 \hat{\eta}_{1}+2 \sqrt{2} 3^{3 / 4} C_{1}+22 \sqrt{2} 3^{1 / 4} \delta_{1}-954 \sqrt{2} 3^{1 / 4} \ln 2+22896 \sqrt{2} 3^{1 / 4}\right. \\
& \left.+8 \sqrt{2} 3^{3 / 4} \delta_{3}+3 \sqrt{2} 3^{1 / 4} \delta_{1} \ln 2\right) \frac{1}{x^{2}}-\frac{3^{1 / 4}}{36} \gamma\left(-9 \sqrt{2} 3^{3 / 4} \delta_{1} \ln 2-4410 \sqrt{2} 3^{3 / 4} \ln 2\right. \\
& \left.+30384 \sqrt{2} 3^{3 / 4}+44 \sqrt{3} \delta_{2}+24 \sqrt{2} 3^{3 / 4} \delta_{1}+6 \sqrt{2} 3^{1 / 4} C_{1}+264 \sqrt{3} \hat{\eta}_{1}+24 \sqrt{2} 3^{1 / 4} \delta_{3}\right) \frac{1}{x} \\
& +\left(8 \sqrt{2} 3^{3 / 4}-\frac{2}{9}\left(-3 \sqrt{2} 3^{3 / 4} \delta_{1}-49975 \sqrt{2} 3^{3 / 4}-216 \sqrt{3} \hat{\eta}_{1}+12 \sqrt{3} \delta_{2}\right) \gamma\right) \ln x+\text { finite. }
\end{aligned}
$$

The residues of all pole singularities in (3.17) vanish provided ${ }^{5}$

$$
\begin{aligned}
\hat{\eta}_{1}= & \frac{3^{1 / 4} \sqrt{2}}{432}\left(7182-15 \delta_{1}+2^{5 / 2} 3^{3 / 4} \delta_{2}\right) \\
C_{1}= & -\frac{\sqrt{2} 3^{3 / 4}}{216}\left(122238 \sqrt{2} 3^{3 / 4}+18 \sqrt{2} 3^{3 / 4} \delta_{1} \ln 2-67 \sqrt{2} 3^{3 / 4} \delta_{1}+64 \sqrt{3} \delta_{2}\right. \\
& \left.-5724 \sqrt{2} 3^{3 / 4} \ln 2+144 \sqrt{2} 3^{1 / 4} \delta_{3}\right) \\
\delta_{1}= & -288, \quad \delta_{2}=-144 \sqrt{2} 3^{1 / 4}
\end{aligned}
$$

where we kept explicit dependence on $\delta_{1}, \delta_{2}$ in $\hat{\eta}_{1}$ and $C_{1}$ (as determined by the vanishing of the residues of the poles in (3.17) up to order three inclusive); the vanishing of the residues of the second order and the first order poles in (3.17) determines $\delta_{1}$ and $\delta_{2}$.

As for the Ricci scalar (3.12), the logarithmic singularity in the Riemann tensor squared at the third order in the late-time expansion, (3.17), remains. This remaining

\footnotetext{
${ }^{5}$ The value of $\hat{\eta}_{1}$ agrees with the one determined from the nonsingularity of the second order late-time curvature invariants in [15].
} 
singularity has both the supergravity piece (in agreement with [24]) and the new $\mathcal{O}(\gamma)$ contribution.

Notice that while $\hat{\eta}_{0}, C_{0}(3.13)$ are determined unambiguously from the nonsingularity condition of the background geometry, the absence of singularities is not a powerful enough constraint to fix $C_{1}{ }^{6}$. This fact will not preclude us from computing a definite value of non-linear second-order hydrodynamic coefficient $\lambda_{1}$.

\section{$3.3 \mathcal{R}_{\mu \nu} \mathcal{R}^{\mu \nu}$ at order $\mathcal{O}\left(\tau^{-2}\right)$}

Analysis of the square of the Ricci tensor can be performed in the same way as for the Riemann tensor squared. We find

$$
\mathcal{R}_{\mu \nu} \mathcal{R}^{\mu \nu}=\cdots+\frac{\gamma}{\tau^{2}}\left\{\frac{4096}{3^{1 / 4}} \sqrt{2} \ln x+\text { finite }-1920 \hat{\alpha}_{3}(x)\right\}, \quad x \equiv 3^{1 / 4}-v \rightarrow 0_{+},
$$

where we explicitly indicated the dependence on order three fields; as before, $\cdots$ indicate lower orders in the late time expansion studied in [15]. We pointed out above that $\hat{\alpha}_{3}(v)$ can be chosen to be finite as $v \rightarrow 3_{-}^{1 / 4}$; this would guarantee the absence of pole singularities in $\mathcal{R}_{\mu \nu} \mathcal{R}^{\mu \nu}$ to orders $\mathcal{O}\left(\tau^{-2}\right)$ and $\mathcal{O}(\gamma)$.

\subsubsection{Higher order curvature invariants}

As in [26] we denote

$$
\mathcal{R}^{\left[2^{n}\right]}{ }_{\mu \nu \rho \lambda} \equiv \mathcal{R}^{\left[2^{n-1}\right]}{ }_{\mu_{1} \nu_{1} \mu \nu} \cdot \mathcal{R}^{\left[2^{n-1}\right] \mu_{1} \nu_{1}}{ }_{\rho \lambda}
$$

where

$$
\mathcal{R}^{[0]}{ }_{\mu \nu \rho \lambda} \equiv \mathcal{R}_{\mu \nu \rho \lambda}
$$

We further define higher curvature invariants $\mathcal{I}^{\left[2^{n}\right]}$, generalizing (3.14):

$$
\begin{aligned}
\mathcal{I}^{\left[2^{n}\right]} & \equiv \mathcal{R}^{\left[2^{n-1}\right]}{ }_{\mu \nu \rho \lambda} \mathcal{R}^{\left[2^{n-1}\right] \mu \nu \rho \lambda} \\
& =\left(\mathcal{I}_{0}^{\left[2^{n}\right] S U G R A}(v)+\gamma \mathcal{I}_{0}^{\left[2^{n}\right] W}(v)\right)+\frac{1}{\tau^{2 / 3}}\left(\mathcal{I}_{1}^{\left[2^{n}\right] S U G R A}(v)+\gamma \mathcal{I}_{1}^{\left[2^{n}\right] W}(v)\right) \\
& +\frac{1}{\tau^{4 / 3}}\left(\mathcal{I}_{2}^{\left[2^{n}\right] S U G R A}(v)+\gamma \mathcal{I}_{2}^{\left[2^{n}\right] W}(v)\right)+\frac{1}{\tau^{2}}\left(\mathcal{I}_{3}^{\left[2^{n}\right] S U G R A}(v)+\gamma \mathcal{I}_{3}^{\left[2^{n}\right] W}(v)\right) \\
& +\mathcal{O}\left(\tau^{-8 / 3}\right)+\mathcal{O}\left(\gamma^{2}\right) .
\end{aligned}
$$

\footnotetext{
${ }^{6}$ Although, unlike the analysis up to the second order [15], $\hat{\eta}_{1}$ is determined unambiguously here.
} 
Given the complexity of the analysis, we checked at order $\mathcal{O}\left(\tau^{-2}\right)$ only the nonsingularity of $\mathcal{I}^{[4]}$. Using the results of the Appendices A and B, as well as (3.13) and (3.18), we find

$$
\mathcal{I}_{3}^{[2] S U G R A}+\left.\gamma \mathcal{I}_{3}^{[2] W}\right|_{x \equiv 3^{1 / 4}-v \rightarrow 0_{+}}=\left(\frac{640}{3^{1 / 4}} \sqrt{2}+\gamma \frac{5636512}{3^{5 / 4}} \sqrt{2}\right) \ln x+\text { finite } .
$$

The supergravity part of the logarithmic singularity in (3.23) agrees with the corresponding computation in [26].

\section{$4 \lambda_{1}$ for the Bjorken flow of $\mathcal{N}=4$ SYM plasma}

In the previous section we analytically evaluated $\alpha^{\prime}$-corrected supergravity background dual to the Bjorken flow of $\mathcal{N}=4 \mathrm{SYM}$ plasma at finite coupling to order $\mathcal{O}\left(\tau^{-2}\right)$ in the late proper time expansion. We can now extract the boundary energy density $\epsilon(\tau)$ from the one-point correlation function of the boundary stress energy tensor using the $\alpha^{\prime}$-corrected holographic renormalization developed in [29]. We confirmed that the final expression for the energy density can be evaluated as in the supergravity approximation $[21,23,26]$ :

$$
\epsilon(\tau)=-\frac{N^{2}}{2 \pi^{2}} \lim _{v \rightarrow 0} \frac{2 a(v, \tau)}{v^{4} \tau^{4 / 3}} .
$$

Using the results of the lower orders in the late proper time expansion [15], the details presented in Appendices A and B, (2.5), (3.13) and (3.18) we find ${ }^{7}$

$$
\begin{aligned}
& \epsilon(\tau)=\frac{N^{2}\left(6+576 \gamma+\gamma \delta_{1}\right)}{12 \pi^{2}} \frac{1}{\tau^{4 / 3}}-\frac{N^{2} 2^{1 / 2} 3^{1 / 4}\left(1566 \gamma+8+\gamma \delta_{1}\right)}{48 \pi^{2}} \frac{1}{\tau^{2}} \\
& +\frac{N^{2} 3^{1 / 2}}{864 \pi^{2}}\left(12+24 \ln 2+\gamma\left(2 \delta_{1} \ln 2+\delta_{1}+7086+4212 \ln 2\right)\right) \frac{1}{\tau^{8 / 3}}+\mathcal{O}\left(\tau^{-10 / 3}\right) .
\end{aligned}
$$

The string theory result (4.2) should now be interpreted within second order relativistic conformal hydrodynamics $[8,9]$. For the Bjorken flow of the $\mathcal{N}=4 \mathrm{SYM}$ plasma we expect [8]

$$
\begin{aligned}
& \frac{\epsilon^{\text {gauge }}(\tau)}{\mathcal{C}}=\tau^{-4 / 3}-2 \eta_{0} \tau^{-2}+\left[\frac{3}{2} \eta_{0}^{2}-\frac{2}{3}\left(\eta_{0} \tau^{\Pi}-\lambda_{1}^{0}\right)\right] \tau^{-8 / 3}+\mathcal{O}\left(\tau^{-10 / 3}\right) \\
& \eta=\mathcal{C} \eta_{0}\left(\frac{\epsilon}{\mathcal{C}}\right)^{3 / 4}, \quad \tau_{\Pi}=\tau_{\Pi}^{0}\left(\frac{\epsilon}{\mathcal{C}}\right)^{-1 / 4}, \quad \lambda_{1}=\mathcal{C} \lambda_{1}^{0}\left(\frac{\epsilon}{\mathcal{C}}\right)^{1 / 2}
\end{aligned}
$$

\footnotetext{
${ }^{7}$ Despite the fact that at order three $\delta_{1}$ and $\delta_{2}$ are fixed (see (3.18)), we keep them arbitrary to compare with [15]. As in [15], the dependence on $\delta_{i}$ disappears in physical quantities.
} 
where $\mathcal{C}$ is an arbitrary scale, related to the initial energy density of the expanding plasma.

To match the string theory result (4.2) with (4.3) we need to recall the equation of state for the $\mathcal{N}=4$ SYM plasma [30]

$$
\epsilon(T)=\frac{3}{8} \pi^{2} N^{2} T^{4}(1+15 \gamma),
$$

and the $\mathcal{N}=4$ SYM relaxation time $\tau_{\Pi}[18]$

$$
\tau_{\Pi} T=\frac{2-\ln 2}{2 \pi}+\frac{375}{4 \pi} \gamma .
$$

Ultimately, we find:

$$
\frac{\eta}{s}=\frac{1}{4 \pi}(1+120 \gamma), \quad \frac{\lambda_{1} T}{\eta}=\frac{1}{2 \pi}(1+215 \gamma) .
$$

Notice that the ratio of shear viscosity to the entropy density agrees with the results reported in $[12,13,14,15]$, and the supergravity part of $\lambda_{1}$ agrees with computations in $[8,9]$.

\section{Computation in the framework of Kinoshita et al}

In this section we compute the finite coupling correction to $\lambda_{1}$ by using the framework of Kinoshita and collaborators [28] for finding the holographic dual of an expanding plasma (see also [27]). We will work with the five-dimensional action

$$
\begin{aligned}
S & =\frac{1}{16 \pi G} \int d^{5} x \sqrt{-g}(R+12+\gamma W), \\
W & \equiv-\frac{1}{4} C_{a b c d} C^{a b}{ }_{e f} C_{g h}{ }^{c e} C^{g h d f}+C_{a b c d} C_{e f}^{a c} C_{g h}^{b}{ }^{b} C^{g d h f},
\end{aligned}
$$

where $C_{a b c d}$ is the five-dimensional Weyl tensor. The use of this action was justified in [20], where it was also shown that it leads to universal finite coupling corrections to hydrodynamic coefficients. The holographic dual to the Bjorken flow of the CFT plasma is taken to be [28] of the form

$$
\begin{aligned}
d s^{2} & =g_{\mu \nu} d x^{\mu} d x^{\nu} \\
& =-r^{2} a d \tau^{2}+2 d \tau d r+r^{2} \tau^{2} e^{2 b-2 c}\left(1+\frac{1}{r t}\right)^{2} d y^{2}+r^{2} e^{c} d x_{\perp}^{2} .
\end{aligned}
$$


Plugging this form for the metric into the Einstein equations of motion, one can find the functions $a(t, r), b(t, r), c(t, r)$ order by order in a late-time expansion as before:

$$
\begin{aligned}
& a(t, r)=a_{0}(v)+u a_{1}(v)+u^{2} a_{2}(v)+\ldots, \\
& b(t, r)=b_{0}(v)+u b_{1}(v)+u^{2} b_{2}(v)+\ldots, \\
& c(t, r)=c_{0}(v)+u c_{1}(v)+u^{2} c_{2}(v)+\ldots,
\end{aligned}
$$

where $v \equiv r t^{1 / 3}, u \equiv t^{-2 / 3}$. In [28] these functions were explicitly computed:

$$
\begin{gathered}
a_{0}(v)=1-\frac{w^{4}}{v^{4}}, \quad b_{0}(v)=c_{0}(v)=0 \\
a_{1}(v)=-\frac{2}{3} \frac{\left(\xi_{1}+1\right) v^{4}-3 M_{1} w^{4} v+w^{4} \xi_{1}}{v^{5}}, \quad b_{1}(v)=-\frac{\xi_{1}+1}{v} \\
c_{1}(v)=-\frac{2 \xi_{1}}{3 v}-\frac{1}{2} M_{1} \ln \left(1-\frac{w^{4}}{v^{4}}\right)+\frac{\arctan \left(\frac{v}{w}\right)+\frac{1}{2} \ln \left(\frac{v-w}{v+w}\right)-\frac{\pi}{2}}{3 w} \\
a_{2}(v)=-\frac{2\left(v^{4}+w^{4}\right) \xi_{2}}{3 v^{5}}-\frac{4\left(v^{3}-3 w^{4} M_{1}\right) \xi_{1}}{9 v^{5}}+\frac{\xi_{1}^{2}\left(v^{4}-3 w^{4}\right)}{9 v^{6}} \\
+\frac{\left(v^{4}+w^{4}\right)\left(9 w^{2} M_{1}^{2}+1\right) \arctan \left(\frac{v}{w}\right)}{6 v^{5} w}-\frac{\left(v^{4}-2 w^{3} v+w^{4}\right)\left(9 w^{2} M_{1}^{2}-1\right) \ln (v-w)}{12 v^{5} w} \\
+\frac{\left(v^{4}+2 w^{3} v+w^{4}\right)\left(9 w^{2} M_{1}^{2}-1\right) \ln (v+w)}{12 v^{5} w}+\frac{\left(9 M_{1}^{2} w^{4}+w^{2}\right) \ln \left(v^{2}+w^{2}\right)}{6 v^{4}} \\
-\frac{3\left(3(12 \ln (v)+5) v M_{1}+4\right) M_{1} w^{4}+4\left(3 v M_{2} w^{4}+v^{3}\right)}{18 v^{5}}, \\
b_{2}(v)=-\frac{\xi_{1}^{2}}{6 v^{2}}+\frac{1}{4} M_{1}\left(-24 M_{1} \ln (v)-\frac{4}{v}+\frac{\pi}{w}\right)+\frac{\left(9 w^{2} \eta_{0}^{2}-2 v M_{1}+1\right) \arctan \left(\frac{v}{w}\right)}{4 v w} \\
+\frac{\left(3 w M_{1}-1\right)\left(2 v-3 w+3 w(4 v-3 w) M_{1}\right) \ln (v-w)}{24 v w^{2}} \\
+\frac{\left(3 w M_{1}+1\right)\left(-2 v-3 w+3 w(4 v+3 w) M_{1}\right) \ln (v+w)}{24 v w^{2}} \\
+\frac{1}{12}\left(18 M_{1}^{2}+\frac{1}{w^{2}}\right) \ln \left(v^{2}+w^{2}\right)-\frac{\xi_{2}}{v}+\frac{1}{2 v^{2}},
\end{gathered}
$$




$$
\begin{aligned}
& c_{2}^{\prime}(v)=\frac{\left(12 w M_{1} v^{5}-6 w v^{4}+\pi\left(v^{4}-w^{4}\right) v+2 w^{5}\right) M_{1} w^{3}}{3\left(v^{5}-v w^{4}\right)^{2}}+\frac{2 \xi_{1}^{2}}{9 v^{3}} \\
& +\frac{4 v^{2} \ln (v) M_{1}}{3\left(v^{4}-w^{4}\right)}+\frac{\left(6\left(w^{4}-5 v^{4}\right) M_{1} w^{4}+4 v^{3}\left(v^{4}+w^{4}\right)\right) \xi_{1}}{9\left(v^{5}-v w^{4}\right)^{2}}+\frac{2 \xi_{2}}{3 v^{2}} \\
& +\frac{\left(v^{4}+3 w^{4}-3 w^{2} M_{1}\left(4 v w^{2}+9\left(v^{4}-w^{4}\right) M_{1}\right)\right) \arctan \left(\frac{v}{w}\right)}{18 v^{2}\left(v^{4}-w^{4}\right) w} \\
& -\frac{\left(\left(3 w M_{1}-1\right)\left((v+w)\left(v^{2}-2 w v+3 w^{2}\right)-9(v-w) w\left(v^{2}+w^{2}\right) M_{1}\right)\right) \ln (v-w)}{36 v^{2}(v-w)\left(v^{2}+w^{2}\right) w} \\
& -\frac{\left(\left(3 w M_{1}+1\right)\left((v-w)\left(v^{2}+2 w v+3 w^{2}\right)+9 w(v+w)\left(v^{2}+w^{2}\right) M_{1}\right)\right) \ln (v+w)}{36 v^{2}(v+w)\left(v^{2}+w^{2}\right) w} \\
& -\frac{\left(3 M_{1} v^{3}+w^{2}\right) \ln \left(v^{2}+w^{2}\right)}{9 v^{5}-9 v w^{4}-3 w\left(4 M_{2} w^{4}+v^{2}\right)}
\end{aligned}
$$

Both $\xi_{1}$ and $\xi_{2}$ are gauge degrees of freedom which can be set to a convenient value. In what follows we take $\xi_{1}=-1$ and leave $\xi_{2}$ arbitrary as a cross-check on our calculations.

As explained in [28], the energy momentum tensor of the plasma can be read off from the function $a(t, r)$, by expanding the function $a(t, r)$ in the large $r$ limit. More concretely,

$$
\begin{aligned}
a(t, r) & =1+\frac{a^{(1)}(\tau)}{r}+\ldots+\frac{a^{(4)}(\tau)}{r^{4}}+\ldots, \\
T_{\tau \tau} & \equiv \epsilon(\tau)=-\frac{3}{2} \frac{N^{2}}{4 \pi^{2}} a^{(4)}(\tau) .
\end{aligned}
$$

Performing this expansion in the above solution to $a(t, r)$ leads to

$$
\epsilon(\tau)=\frac{3}{2} \frac{N^{2}}{4 \pi^{2}} \omega^{4}\left(\tau^{-4 / 3}-2 M_{1} \tau^{2}+\left(\frac{9 M_{1}^{2}+4 M_{2}}{6}\right) \tau^{-8 / 3}\right) .
$$

This is to be equated with the hydrodynamic expansion for $\epsilon(\tau)$,

$$
\frac{\epsilon(\tau)}{\epsilon_{0}}=\tau^{-4 / 3}-2 \eta_{0} \tau^{-2}+\frac{9 \eta_{0}^{2}+4\left(\lambda_{1}^{0}-\eta_{0} \tau_{\Pi}^{0}\right)}{6} \tau^{-8 / 3},
$$

leading to the identifications

$$
\epsilon_{0}=\frac{3}{2} \frac{N^{2}}{4 \pi^{2}} \omega^{4}, \quad M_{1}=\eta_{0}, \quad M_{2}=\lambda_{1}^{0}-\eta_{0} \tau_{\Pi}^{0} .
$$

These constants are in turn fixed by imposing regularity of the function $c(t, r)[28]$. An expansion of this function around $v=w$ has a simple pole, unless we set

$$
M_{1}=\frac{1}{3 w}, \quad M_{2}=\frac{\ln 2-1}{6 w^{2}} .
$$


Finally, the hydrodynamic coefficients are obtained by the relations

$$
\eta=\epsilon_{0} \eta_{0}\left(\frac{\epsilon}{\epsilon_{0}}\right)^{3 / 4}, \quad \tau_{\Pi}=\tau_{\Pi}^{0}\left(\frac{\epsilon}{\epsilon_{0}}\right)^{-1 / 4}, \quad \lambda_{1}=\epsilon_{0} \lambda_{1}^{0}\left(\frac{\epsilon}{\epsilon_{0}}\right)^{1 / 2} .
$$

We now want to compute $\gamma$ corrections to these by using the $\gamma$ corrected action (5.2). Since it is not practical to find the equations of motions from the action (5.2), we will work in an effective action framework, as in [15]. To do this, first notice that in the metric (5.3) there are two implicit constraints, namely

$$
g_{r r}=0, \quad g_{\tau r}=1 .
$$

It is not correct to impose these constraints at the level of the action. They should only be imposed on the equations of motion. Therefore we modify the metric (5.3) to:

$$
\begin{aligned}
d s^{2}= & g_{\mu \nu} d x^{\mu} d x^{\nu} \\
= & -r^{2} a(t, r) d \tau^{2}+2\left(1+h(t, r)-\frac{1}{2} a(t, r) g(t, r)\right) d \tau d r+\frac{g(t, r)}{r^{2}} d r^{2} \\
& +r^{2} \tau^{2} e^{2 b(t, r)-2 c(t, r)}\left(1+\frac{1}{r t}\right)^{2} d y^{2}+r^{2} e^{c}\left(d x_{1}^{2}+d x_{2}^{2}\right) .
\end{aligned}
$$

We further substitute

$$
\begin{aligned}
& a(t, r)=a_{0}(v)+u a_{1}(v)+u^{2} a_{2}(v)+\gamma \hat{a}(t, r), \\
& b(t, r)=b_{0}(v)+u b_{1}(v)+u^{2} b_{2}(v)+\gamma \hat{b}(t, r), \\
& c(t, r)=c_{0}(v)+u c_{1}(v)+u^{2} c_{2}(v)+\gamma \hat{c}(t, r,
\end{aligned}
$$

and evaluate the action (5.2) on the modified metric. A few comments are in order:

- One only needs to evaluate the action to linear order in $g, h$.

- It is sufficient to compute $W$ to linear order with respect to each and every field $\hat{a}, \hat{b}, \hat{c}, g, h$, meaning no mixed terms such as $\hat{a} \hat{b}, g \hat{c}$ can appear in $W$.

- Since we are interested in computing $\hat{a}, \hat{b}, \hat{c}$ to quadratic order in $u$, it is sufficient to evaluate $W$ to linear order in $a_{2}, b_{2}, c_{2}$ and quadratic order in $a_{1}, b_{1}, c_{1}$.

Variation of the action $S=S(\hat{a}, \hat{b}, \hat{c}, g, h)$ with respect to the various fields lead to the equations of motion

$$
\frac{\delta S}{\delta \hat{a}(t, r)_{\mid g, h=0}}=0, \quad \frac{\delta S}{\delta \hat{b}(t, r)_{\mid g, h=0}}=0, \quad \frac{\delta S}{\delta \hat{c}(t, r)}_{\mid g, h=0}=0,
$$


and to the constraints

$$
\frac{\delta S}{\delta g(t, r)}_{\mid g, h=0}=0, \quad \frac{\delta S}{\delta h(t, r)}_{\mid g, h=0}=0 .
$$

After finding these equations, one expands $\hat{a}, \hat{b}, \hat{c}$ to quadratic order in $u$, exactly as it was done for the unhatted quantities. This leads to a set of equations for the hatted quantities $\hat{a}_{i}, \hat{b}_{i}, \hat{c}_{i}, i=0,1,2$.

\subsection{Order - 0}

It will be convenient to perform the change of variable $y \equiv w / v$. In terms of this coordinate the equations of motion are

$$
\begin{aligned}
\hat{b}_{0}^{\prime \prime} & =S_{B}^{0}, \\
\left(\hat{c}_{0}^{\prime \prime}-\frac{2}{3} \hat{b}_{0}^{\prime \prime}\right)-\frac{3+y^{4}}{y\left(1-y^{4}\right)}\left(\hat{c}_{0}^{\prime}-\frac{2}{3} \hat{b}_{0}^{\prime}\right) & =S_{C}^{0}, \\
\hat{a}_{0}^{\prime \prime}-\frac{6 \hat{a}_{0}^{\prime}}{y}+\frac{12 \hat{a}_{0}}{y^{2}}-\frac{2\left(y^{4}+3\right) \hat{c}_{0}^{\prime}}{y}+2\left(1-y^{4}\right) \hat{c}_{0}^{\prime \prime} & =S_{A}^{0} .
\end{aligned}
$$

The solution of this system of equations is straightforward. Imposing the boundary conditions $\hat{a}_{0}(0)=\hat{b}_{0}(0)=\hat{c}_{0}(0)=0$ leads to

$$
\begin{aligned}
& a_{0}(y)=A_{1}^{0} y^{4}+A_{2}^{0} y^{3}+\frac{15}{11} y^{12}\left(72-49 y^{4}\right)-\frac{2}{3} B_{1}^{0} y\left(1+y^{4}\right), \\
& b_{0}(y)=-\frac{360}{11} y^{12}-B_{1}^{0} y, \\
& c_{0}(y)=-\frac{2}{3} B_{1}^{0} y-\frac{240}{11} y^{12}+C_{1}^{0} \ln \left(1-y^{4}\right) .
\end{aligned}
$$

The coefficient $B_{1}^{0}$ is a gauge parameter [28] and we will set it to zero in what follows. The constraint equations further impose $A_{2}^{0}=C_{1}^{0}=0$. Quite generally, the constraint equations can only affect the coefficients of the solutions to the homogeneous equations, and since these come from the supergravity part of the action, $C^{4}$ should not alter the constraints from those at the supergravity level. Another way to see this is to note that one can always expand the equations of motion about $y=0$, where all the source terms are negligible. The constraint equations must necessarily be the same around $y=0$ as at any other point, as they only fix constants.

Expanding $a_{0}(y)$ around $y=0$ and taking the coefficient of $y^{4}$ we see that this solution modifies $\epsilon_{0}$ at order $\gamma$ by some undetermined constant $A_{1}^{0}$, which will however not affect any of the physical results as we will see. 


\subsection{Order -1}

The equations of motion are exactly the same as before (5.17), performing the replacement $0 \rightarrow 1$ in the indices of functions and sources. The solutions are now:

$$
\begin{gathered}
w \hat{a}_{1}(y)=A_{1}^{1} y^{4}+A_{2}^{1} y^{3}-\frac{10\left(-343 y^{5}-196 y^{4}+360 y+216\right) y^{12}}{11} \\
-\frac{2\left(A_{0}^{1} y^{5}+B_{1}^{1}\left(1+y^{4}\right) y\right)}{3}, \\
w \hat{b}_{1}(y)=-B_{1}^{1} y+\frac{720 y^{12}(2 y+1)}{11}, \\
w \hat{c}_{1}(y)=\frac{960 y^{13}}{11}+\frac{2873 y^{12}}{66}-\frac{490 y^{9}}{99}+\frac{335 y^{8}}{22}-\frac{52 y^{5}}{11}+\frac{555 y^{4}}{22} \\
+\frac{1}{792}\left(-528 B_{1}^{1}-2160\right) y+\frac{1}{792}\left(66\left(A_{1}^{0}+15\right) \arctan \left(\frac{1}{y}\right)-33\left(A_{1}^{0}+15\right) \pi\right) \\
+\frac{\left(11 A_{1}^{0}+345\right) y\left(y^{2}+y+1\right)}{66\left(y^{3}+y^{2}+y+1\right)} \\
-\frac{\left(15+A_{1}^{0}\right) \operatorname{arctanh}(y)+\left(2 A_{1}^{0}-3\left(80+C_{1}^{1}\right)\right) \ln \left(1-y^{4}\right)}{12} .
\end{gathered}
$$

The constraint equations now imply $A_{2}^{1}=A_{1}^{1}+C_{1}^{1}=0$. We may further set $B_{1}^{1}$ to zero as we did at order zero. The constant $C_{1}^{1}$ is determined by imposing regularity at $y=1$ (that is, $v=w$ ). The function $\hat{c}_{1}(y)$ has a pole there unless we set $C_{1}^{1}=\left(A_{1}^{0}-165\right) / 2$.

Expanding $a_{1}(y)$ around $y=0$ and taking the coefficient of $y^{4}$ we get a non-zero contribution. This implies that to this order the energy density is now given by

$$
\epsilon(\tau)=\frac{3}{2} \frac{N^{2}}{4 \pi^{2}} w^{4}\left(\left(1-A_{1}^{0} \gamma\right) \tau^{-4 / 3}-2\left(M_{1}+\gamma \frac{A_{1}^{0}-165}{4 w}\right) \tau^{2}\right),
$$

with $M_{1}$ given by (5.11). Now, in the late time regime we assume that the expressions for the energy and entropy densities are given by

$$
\epsilon=\frac{3}{8} \pi^{2} N^{2} T^{4}(1+15 \gamma), \quad s=\frac{\pi^{2}}{2} N^{2} T^{3}(1+15 \gamma)
$$

Then, this result together with (5.12) leads to

$$
\frac{\eta}{s}=\frac{1}{4 \pi}(1+120 \gamma)
$$

in perfect agreement with what was previously known in the literature $[12,13,14,15]$. Notice the undetermined constant $A_{1}^{0}$ has canceled out of the result. 


\subsection{Order -2}

The equations of motion are as in the previous sections, but now using the source terms of order 2. These are too long to display here, but can be obtained from the authors at request. For small $y$ all source terms vanish and so we get only the homogeneous equations solutions, namely

$$
\begin{array}{r}
w^{2} \hat{a}_{2}(y)=A_{1}^{2} y^{4}+A_{2}^{2} y^{3}, \\
w^{2} b_{2}(y)=-B_{1}^{2} y, \\
w^{3} c_{2}^{\prime}(y)=C_{1}^{2} y^{5} .
\end{array}
$$

One may once again choose $B_{1}^{2}=0$. The constraint equations force $A_{2}^{2}=2 A_{1}^{2}+C_{1}^{2}=0$. Therefore to determine $A_{1}^{2}$, which is the parameter that enters the energy density expansion, we must find out the value of $C_{1}^{2}$. This can be determined by imposing regularity of the full solution at $y=1$. Close to $y=1$ one has

$$
c_{2}^{\prime}(y)=\frac{(1+2 \ln 2) A_{1}^{0}+18 C_{1}^{2}-510 \ln 2-1085}{72(1-y)}+(\text { Regular at } y=1) .
$$

Imposing regularity forces us to pick

$$
C_{1}^{2}=\frac{1085+510 \ln 2-A_{1}^{0}(1+\ln 4)}{18}=-2 A_{1}^{2} .
$$

The energy density expansion coefficient of $\tau^{-8 / 3}$ receives a modification, namely

$$
\frac{9 \eta_{0}^{2}+4\left(\lambda_{1}^{0}-\eta_{0} \tau_{\Pi}^{0}\right)}{6}=\frac{9 M_{1}^{2}+4 M_{2}}{6}-\gamma A_{1}^{2} .
$$

Then, using (5.12) and the known result [18]

$$
\tau_{\Pi} T=\frac{2-\ln 2}{2 \pi}+\frac{375}{4 \pi} \gamma
$$

together with the expression for $\eta / s$ found in the previous section, leads to

$$
\frac{\lambda_{1} T}{\eta}=\frac{1}{2 \pi}(1+215 \gamma)
$$

in agreement with (4.6). 


\section{Acknowledgments}

We would like to thank Shin Nakamura for valuable comments. Research at Perimeter

Institute is supported by the Government of Canada through Industry Canada and by the Province of Ontario through the Ministry of Research \& Innovation. AB gratefully acknowledges further support by an NSERC Discovery grant and support through the Early Researcher Award program by the Province of Ontario. MP is supported by the Portuguese Fundacao para a Ciencia e Tecnologia, grant SFRH/BD/23438/2005.

\section{A $\quad\left\{a_{2}, b_{2}, c_{2}\right\}$ and $a_{3}$}

Explicit analytic solutions for $\left\{a_{2}, b_{2}, c_{2}\right\}$ were found in [24]:

$$
\begin{aligned}
a_{2}= & \frac{\left(9+5 v^{4}\right) v^{2}}{12\left(9-v^{8}\right)}-C \frac{\left(9+v^{4}\right) v^{4}}{72\left(9-v^{8}\right)}+\hat{\eta}^{2} \frac{\left(-1053-171 v^{4}+9 v^{8}+7 v^{12}\right) v^{4}}{6\left(9-v^{8}\right)^{2}} \\
& +\frac{1}{8 \sqrt{3}} \ln \frac{\sqrt{3}-v^{2}}{\sqrt{3}+v^{2}}-\frac{3}{4} \hat{\eta}^{2} \ln \frac{3-v^{4}}{3+v^{4}}, \\
c_{2}= & -\frac{\pi^{2}}{288 \sqrt{3}}+\frac{v^{2}\left(9+v^{4}\right)}{12\left(9-v^{8}\right)}+C \frac{v^{4}}{72\left(3+v^{4}\right)}-\hat{\eta}^{2} \frac{\left(-9+54 v^{4}+7 v^{8}\right) v^{4}}{6\left(3+v^{4}\right)\left(9-v^{8}\right)} \\
& +\frac{1}{8 \sqrt{3}} \ln \frac{\sqrt{3}-v^{2}}{\sqrt{3}+v^{2}}+\frac{1}{72}\left(C+66 \hat{\eta}^{2}\right) \ln \frac{3-v^{4}}{3+v^{4}} \\
& +\frac{1}{24 \sqrt{3}}\left(\ln \frac{\sqrt{3}-v^{2}}{\sqrt{3}+v^{2}} \ln \frac{\left(\sqrt{3}-v^{2}\right)\left(\sqrt{3}+v^{2}\right)^{3}}{4\left(3+v^{4}\right)^{2}}-\operatorname{li}_{2}\left(-\frac{\left(\sqrt{3}-v^{2}\right)^{2}}{\left(\sqrt{3}+v^{2}\right)^{2}}\right)\right), \\
b_{2}= & -2 c_{2}+\frac{v^{2}}{4\left(3+v^{4}\right)}+C \frac{v^{4}}{24\left(3+v^{4}\right)}+\hat{\eta}^{2} \frac{\left(39+7 v^{4}\right) v^{4}}{2\left(3+v^{4}\right)^{2}}+\frac{1}{8 \sqrt{3}} \ln \frac{\sqrt{3}-v^{2}}{\sqrt{3}+v^{2}} \\
& +\frac{3}{4} \hat{\eta}^{2} \ln \frac{3-v^{4}}{3+v^{4}},
\end{aligned}
$$

where $\{\hat{\eta}, C\}$ are arbitrary parameters.

At third order (for the warp factors $\left\{a_{3}, b_{3}, c_{3}\right\}$ ) one obtains four second order ODE's and a first order constraint, all linear in $\left\{a_{3}, b_{3}, c_{3}\right\}$ (and their derivatives). The two additional equations are the constraints used to fix the radial coordinate and the late time in the boost-invariant metric ansatz (2.2). It is straightforward to use these constraints to solve algebraically for $b_{3}^{\prime}$ and $c_{3}^{\prime}$ in terms of $a_{3}^{\prime}$ and the lower order warp factors. These expressions can further be used to obtain a decoupled second order ODE for $a_{3}$ :

$$
0=a_{3}^{\prime \prime}+\frac{5 v^{16}+18 v^{12}+216 v^{8}+126 v^{4}+243}{\left(v^{8}-9\right) v\left(v^{4}+2 v^{2}+3\right)\left(v^{4}-2 v^{2}+3\right)} a_{3}^{\prime}+\mathcal{J}_{a_{3}},
$$




$$
\begin{aligned}
\mathcal{J}_{a_{3}}= & \frac{2 \hat{\eta}\left(5 v^{12}+9 v^{8}+27 v^{4}+135\right)}{3\left(v^{4}-3\right)^{2}\left(v^{4}+2 v^{2}+3\right)\left(v^{4}-2 v^{2}+3\right)} \ln \frac{3-v^{4}}{3+v^{4}} \\
& +\frac{32 \sqrt{3} v^{6} \hat{\eta}\left(v^{8}+18 v^{4}+9\right)}{\left(v^{8}-9\right)^{2}\left(v^{4}+2 v^{2}+3\right)\left(v^{4}-2 v^{2}+3\right)} \operatorname{arctanh} \frac{v^{2}}{\sqrt{3}} \\
& -\frac{4 \hat{\eta} v^{4}}{3\left(v^{4}-2 v^{2}+3\right)\left(v^{4}+2 v^{2}+3\right)\left(v^{8}-9\right)^{5}}\left(295245+393660 v^{4}-531 v^{32}\right. \\
& -2556 v^{28}-9882 v^{24}-21708 v^{20}+27702 v^{16}+358668 v^{12}+898857 v^{8}+v^{40} \\
& -42768 \hat{\eta}^{2} v^{26}-1113264 v^{22} \hat{\eta}^{2}-2554416 \hat{\eta}^{2} v^{18}-7336656 v^{14} \hat{\eta}^{2}-12912048 \hat{\eta}^{2} v^{10} \\
& -46294416 \hat{\eta}^{2} v^{6}-9447840 \hat{\eta}^{2} v^{2}+432 v^{30} C+12 v^{34} C-52488 v^{10} C+27216 v^{14} C \\
& \left.-78732 v^{2} C-3024 v^{22} C+1296 v^{30} \hat{\eta}^{2}+144 v^{34} \hat{\eta}^{2}-314928 v^{6} C+648 v^{26} C\right) .
\end{aligned}
$$

For the computation of $C$ we actually need only the asymptotic solution of $a_{3}^{\prime}(v)$ as $x \equiv 3^{1 / 4}-v \rightarrow 0_{+}$. Using (A.2) and (A.3) we find:

$$
\left.\frac{d a_{3}(v)}{d v}\right|_{x \equiv 3^{1 / 4}-v \rightarrow 0_{+}}=\frac{\hat{\eta}^{3} 3^{3 / 4}}{8} \frac{1}{x^{4}}+\left(\frac{11 \hat{\eta}^{3} \sqrt{3}}{24}-\frac{\hat{\eta}}{12}+\frac{\sqrt{3} \hat{\eta} C}{144}\right) \frac{1}{x^{3}}+\mathcal{A}_{3} \frac{1}{x^{2}}+\mathcal{O}\left(x^{-1}\right)
$$

where $\mathcal{A}_{3}$ must be fixed so it satisfies the boundary condition (3.1).

\section{B Data for computing $\mathcal{I}_{3}^{[2] W}$}

The coefficients $\mathcal{Q}_{i}$ in (3.16) are given by:

$$
\begin{aligned}
& \mathcal{Q}_{1}=102036672+177147 \delta_{1}+\left(-177147 \delta_{1}-102036672\right) v^{4}+\left(-452709 \delta_{1}\right. \\
& -8039875644) v^{8}+\left(-229635 \delta_{1}+17670478860\right) v^{12}+\left(4374 \delta_{1}-10087292556\right) v^{16} \\
& +\left(2301024348+42282 \delta_{1}\right) v^{20}+\left(14094 \delta_{1}-758889972\right) v^{24}+\left(373696740+162 \delta_{1}\right) v^{28} \\
& -\left(945 \delta_{1}+73262340\right) v^{32}+\left(-207 \delta_{1}+3556980\right) v^{36}-9 v^{40} \delta_{1}+v^{44} \delta_{1},
\end{aligned}
$$




$$
\begin{aligned}
& \mathcal{Q}_{2}=-258280326 \hat{\eta}_{0} \delta_{1}-74384733888 \hat{\eta}_{0}+43046721 \delta_{2}+\left(-86093442 \hat{\eta}_{0} \delta_{1}\right. \\
& \left.+41324852160 \hat{\eta}_{0}+52612659 \delta_{2}\right) v^{4}+\left(-1004423490 \hat{\eta}_{0} \delta_{1}+9228872608632 \hat{\eta}_{0}\right. \\
& \left.+113196933 \delta_{2}\right) v^{8}+\left(158192271 \delta_{2}-1491223446 \hat{\eta}_{0} \delta_{1}-33853663263240 \hat{\eta}_{0}\right) v^{12} \\
& +\left(105323733 \delta_{2}-798224382 \hat{\eta}_{0} \delta_{1}+69144574698756 \hat{\eta}_{0}\right) v^{16}-\left(38887909614036 \hat{\eta}_{0}\right. \\
& \left.-32483511 \delta_{2}+170809074 \hat{\eta}_{0} \delta_{1}\right) v^{20}-\left(14604786 \hat{\eta}_{0} \delta_{1} 273375 \delta_{2}\right. \\
& \left.-22636564766388 \hat{\eta}_{0}\right) v^{24}-\left(4704237 \delta_{2}+12251574 \hat{\eta}_{0} \delta_{1}+5034336682644 \hat{\eta}_{0}\right) v^{28} \\
& -\left(2229525 \delta_{2}+5466042 \hat{\eta}_{0} \delta_{1}-4161674125224 \hat{\eta}_{0}\right) v^{32}-\left(568215 \delta_{2}+906940950984 \hat{\eta}_{0}\right. \\
& \left.-1465290 \hat{\eta}_{0} \delta_{1}\right) v^{36}+\left(371486277216 \hat{\eta}_{0}-76545 \delta_{2}+1625994 \hat{\eta}_{0} \delta_{1}\right) v^{40}-\left(49680556848 \hat{\eta}_{0}\right. \\
& \left.-6813 \delta_{2}-451278 \hat{\eta}_{0} \delta_{1}\right) v^{44}+\left(47142 \hat{\eta}_{0} \delta_{1}+18898470612 \hat{\eta}_{0}+9399 \delta_{2}\right) v^{48}+\left(3821 \delta_{2}\right. \\
& \left.-1110 \hat{\eta}_{0} \delta_{1}-2382365412 \hat{\eta}_{0}\right) v^{52}+\left(-342 \hat{\eta}_{0} \delta_{1}+923 \delta_{2}+258200244 \hat{\eta}_{0}\right) v^{56}+\left(129 \delta_{2}\right. \\
& \left.-4642164 \hat{\eta}_{0}+126 \hat{\eta}_{0} \delta_{1}\right) v^{60}+\left(8 \delta_{2}+24 \hat{\eta}_{0} \delta_{1}\right) v^{64} \\
& \mathcal{Q}_{3}=-387420489 \hat{\eta}_{0} \delta_{1}-148769467776 \hat{\eta}_{0}+43046721 \delta_{2}+\left(-1607077584 \hat{\eta}_{0} \delta_{1}\right. \\
& \left.+153055008 \delta_{2}-661197634560 \hat{\eta}_{0}\right) v^{4}+\left(2210707403676 \hat{\eta}_{0}-3386342052 \hat{\eta}_{0} \delta_{1}\right. \\
& \left.+267846264 \delta_{2}\right) v^{8}+\left(10723033860480 \hat{\eta}_{0}+232416864 \delta_{2}-3409725456 \hat{\eta}_{0} \delta_{1}\right) v^{12} \\
& +\left(-2092224168 \hat{\eta}_{0} \delta_{1}+103060188 \delta_{2}+18352430617176 \hat{\eta}_{0}\right) v^{16}+\left(18685728 \delta_{2}\right. \\
& \left.-6136825576320 \hat{\eta}_{0}-970923024 \hat{\eta}_{0} \delta_{1}\right) v^{20}+\left(15039362941668 \hat{\eta}_{0}-4846392 \delta_{2}\right. \\
& \left.-352115748 \hat{\eta}_{0} \delta_{1}\right) v^{24}+\left(-1235549044224 \hat{\eta}_{0}-5155488 \delta_{2}-74427984 \hat{\eta}_{0} \delta_{1}\right) v^{28} \\
& +\left(2004210714960 \hat{\eta}_{0}+6647022 \hat{\eta}_{0} \delta_{1}-2215674 \delta_{2}\right) v^{32}+\left(11706768 \hat{\eta}_{0} \delta_{1}\right. \\
& \left.-131529982464 \hat{\eta}_{0}-572832 \delta_{2}\right) v^{36}+\left(4706100 \hat{\eta}_{0} \delta_{1}+188278471332 \hat{\eta}_{0}-59832 \delta_{2}\right) v^{40} \\
& +\left(25632 \delta_{2}-7695285120 \hat{\eta}_{0}+1178064 \hat{\eta}_{0} \delta_{1}\right) v^{44}+\left(15708 \delta_{2}+224640 \hat{\eta}_{0} \delta_{1}\right. \\
& \left.+2953736280 \hat{\eta}_{0}\right) v^{48}+\left(34128 \hat{\eta}_{0} \delta_{1}+3936 \delta_{2}+208054656 \hat{\eta}_{0}\right) v^{52}+\left(3348 \hat{\eta}_{0} \delta_{1}+504 \delta_{2}\right. \\
& \left.+6959196 \hat{\eta}_{0}\right) v^{56}+\left(144 \hat{\eta}_{0} \delta_{1}+32 \delta_{2}\right) v^{60}+\left(\delta_{2}+3 \hat{\eta}_{0} \delta_{1}\right) v^{64}
\end{aligned}
$$


$\mathcal{Q}_{4}=\left(6025163444928 \hat{\eta}_{0}^{3}+69735688020 \hat{\eta}_{0}^{3} \delta_{1}-13947137604 \hat{\eta}_{0}^{2} \delta_{2}\right) v^{2}-278942752080 \hat{\eta}_{0}$

$+\left(539289320688 \hat{\eta}_{0}^{3} \delta_{1}-86782189536 \hat{\eta}_{0}^{2} \delta_{2}-301239576062928 \hat{\eta}_{0}^{3}\right) v^{10}-645700815 \delta_{2}$

$+3874204890 \hat{\eta}_{0} \delta_{1}+\left(378122397264 \hat{\eta}_{0}-1822311189 \delta_{2}+12957063021 \hat{\eta}_{0} \delta_{1}\right) v^{4}$

$+\left(34044733308852 \hat{\eta}_{0}-2368101096 \delta_{2}+29635275924 \hat{\eta}_{0} \delta_{1}\right) v^{12}+\left(-163296 \hat{\eta}_{0}^{2} \delta_{2}\right.$

$\left.-869166288 \hat{\eta}_{0}^{3}-34992 \hat{\eta}_{0}^{3} \delta_{1}\right) v^{58}+\left(518856477120 \hat{\eta}_{0}^{3} \delta_{1}-75303063936 \hat{\eta}_{0}^{2} \delta_{2}\right.$

$\left.-1212305862965760 \hat{\eta}_{0}^{3}\right) v^{14}+\left(6585380676 \hat{\eta}_{0} \delta_{1}-63131883819804 \hat{\eta}_{0}-83613384 \delta_{2}\right) v^{24}$

$+\left(-1405753115292 \hat{\eta}_{0}-73136196 \hat{\eta}_{0} \delta_{1}+2163672 \delta_{2}\right) v^{40}+\left(-7464960 \hat{\eta}_{0}^{3}+5184 \hat{\eta}_{0}^{3} \delta_{1}\right.$

$\left.-10368 \hat{\eta}_{0}^{2} \delta_{2}\right) v^{62}-\left(6931186200 \hat{\eta}_{0}+76284 \delta_{2}+1076544 \hat{\eta}_{0} \delta_{1}\right) v^{52}-\left(49589822592 \hat{\eta}_{0}^{2} \delta_{2}\right.$

$\left.-272744024256 \hat{\eta}_{0}^{3} \delta_{1}-35704672266240 \hat{\eta}_{0}^{3}\right) v^{6}+\left(-1501497972 \delta_{2}-122924025168840 \hat{\eta}_{0}\right.$

$\left.+25694109468 \hat{\eta}_{0} \delta_{1}\right) v^{16}+\left(4697466048 \hat{\eta}_{0}^{3} \delta_{1}+1670378112 \hat{\eta}_{0}^{2} \delta_{2}+134641969016832 \hat{\eta}_{0}^{3}\right)$

$\times v^{30}-\left(5 \delta_{2}+15 \hat{\eta}_{0} \delta_{1}+15120 \hat{\eta}_{0}\right) v^{68}-\left(1275264 \hat{\eta}_{0}^{2} \delta_{2}+23388092928 \hat{\eta}_{0}^{3}+1135296 \hat{\eta}_{0}^{3} \delta_{1}\right)$

$\times v^{54}+\left(-5089392 \hat{\eta}_{0}^{2} \delta_{2}-322667880480 \hat{\eta}_{0}^{3}-14082336 \hat{\eta}_{0}^{3} \delta_{1}\right) v^{50}+\left(292743211968 \hat{\eta}_{0}^{3} \delta_{1}\right.$

$\left.-33391500912 \hat{\eta}_{0}^{2} \delta_{2}-2006104387311648 \hat{\eta}_{0}^{3}\right) v^{18}+\left(-127 \delta_{2}-384048 \hat{\eta}_{0}-522 \hat{\eta}_{0} \delta_{1}\right) v^{64}$

$+\left(-58217292 \hat{\eta}_{0}-10116 \hat{\eta}_{0} \delta_{1}-1608 \delta_{2}\right) v^{60}+\left(-6054175872 \hat{\eta}_{0}^{2} \delta_{2}+116968038336 \hat{\eta}_{0}^{3} \delta_{1}\right.$

$\left.+658418155614720 \hat{\eta}_{0}^{3}\right) v^{22}+\left(44654166 \delta_{2}+183812976 \hat{\eta}_{0} \delta_{1}-16291341648432 \hat{\eta}_{0}\right) v^{32}$

$+\left(15604524936 \hat{\eta}_{0} \delta_{1}-596867292 \delta_{2}-119986465909464 \hat{\eta}_{0}\right) v^{20}+\left(19385568 \hat{\eta}_{0}^{2} \delta_{2}\right.$

$\left.-20320117294896 \hat{\eta}_{0}^{3}-547029936 \hat{\eta}_{0}^{3} \delta_{1}\right) v^{42}+\left(185597568 \hat{\eta}_{0}^{2} \delta_{2}-1635526080 \hat{\eta}_{0}^{3} \delta_{1}\right.$

$\left.+14338868355072 \hat{\eta}_{0}^{3}\right) v^{38}+\left(-6316380 \hat{\eta}_{0} \delta_{1}-272916 \delta_{2}-58737530376 \hat{\eta}_{0}\right) v^{48}$

$-\left(26068068 \hat{\eta}_{0} \delta_{1}+275114490228 \hat{\eta}_{0}+344088 \delta_{2}\right) v^{44}-\left(110621376 \hat{\eta}_{0}^{3} \delta_{1}+8304768 \hat{\eta}_{0}^{2} \delta_{2}\right.$

$\left.-825111360000 \hat{\eta}_{0}^{3}\right) v^{46}+\left(-36868394133108 \hat{\eta}_{0}+1799419860 \hat{\eta}_{0} \delta_{1}+58419144 \delta_{2}\right) v^{28}$

$+\left(14884722 \delta_{2}-105925158 \hat{\eta}_{0} \delta_{1}-5478599707344 \hat{\eta}_{0}\right) v^{36}+\left(-2153635128 \hat{\eta}_{0}^{3} \delta_{1}\right.$

$\left.+717878376 \hat{\eta}_{0}^{2} \delta_{2}-215937884784960 \hat{\eta}_{0}^{3}\right) v^{34}+\left(34888038768 \hat{\eta}_{0}^{3} \delta_{1}\right.$

$\left.-1623120631374384 \hat{\eta}_{0}^{3}+1570231008 \hat{\eta}_{0}^{2} \delta_{2}\right) v^{26}+\left(-233280 \hat{\eta}_{0}^{3}-324 \hat{\eta}_{0}^{2} \delta_{2}+324 \hat{\eta}_{0}^{3} \delta_{1}\right) v^{66}$

$+\left(107373276 \hat{\eta}_{0}-13368 \delta_{2}-127188 \hat{\eta}_{0} \delta_{1}\right) v^{56}+\left(-2563671384 \delta_{2}+23819185620 \hat{\eta}_{0} \delta_{1}\right.$

$\left.-81312328791972 \hat{\eta}_{0}\right) v^{8}$, 


$$
\begin{aligned}
& \mathcal{Q}_{5}=5782609521 v^{4}+3314566899 v^{24}+6632626437 v^{20}+10567349991 v^{16} \\
& +12018183921 v^{12}+9093486951 v^{8}+1375999893 v^{28}+26344593252 \hat{\eta}_{0}^{2} v^{2} \\
& -146014477632 \hat{\eta}_{0}^{2} v^{6}+10005971148 \hat{\eta}_{0}^{2} v^{10}-59066478504 v^{14} \hat{\eta}_{0}^{2}-75754434492 \hat{\eta}_{0}^{2} v^{18} \\
& -55948533840 v^{22} \hat{\eta}_{0}^{2}-22400959860 \hat{\eta}_{0}^{2} v^{26}+26907093 v^{40}+4639059 v^{44}+126080307 v^{36} \\
& +467481699 v^{32}-25 v^{60}+624309 v^{48}+58263 v^{52}+v^{64}+2961 v^{56}-2708022132 v^{34} \hat{\eta}_{0}^{2} \\
& -740454048 v^{38} \hat{\eta}_{0}^{2}-108490428 v^{42} \hat{\eta}_{0}^{2}-16471512 v^{46} \hat{\eta}_{0}^{2}-662580 v^{50} \hat{\eta}_{0}^{2} \\
& -9226708056 v^{30} \hat{\eta}_{0}^{2}-92880 v^{54} \hat{\eta}_{0}^{2}+216 v^{62} \hat{\eta}_{0}^{2}+2916 v^{58} \hat{\eta}_{0}^{2}+2754990144,
\end{aligned}
$$

$$
\begin{aligned}
& \mathcal{Q}_{6}=-12223143-20785248 v^{4}-2196720 v^{24}-6722352 v^{20}-15363675 v^{16} \\
& -23024736 v^{12}-23225940 v^{8}-579312 v^{28}-172186884 \hat{\eta}_{0}^{2} v^{2}+752520456 \hat{\eta}_{0}^{2} v^{6} \\
& -108413964 \hat{\eta}_{0}^{2} v^{10}+166754376 v^{14} \hat{\eta}_{0}^{2}+257348664 \hat{\eta}_{0}^{2} v^{18}+140702832 v^{22} \hat{\eta}_{0}^{2} \\
& +37144008 \hat{\eta}_{0}^{2} v^{26}-828 v^{40}+16 v^{44}-14640 v^{36}-114813 v^{32}-v^{48}+2349324 v^{34} \hat{\eta}_{0}^{2} \\
& +425736 v^{38} \hat{\eta}_{0}^{2}+15876 v^{42} \hat{\eta}_{0}^{2}+648 v^{46} \hat{\eta}_{0}^{2}+12227760 v^{30} \hat{\eta}_{0}^{2}
\end{aligned}
$$

$$
\begin{aligned}
& \mathcal{Q}_{7}=163046532875760 v^{32}-108446607008910 v^{36}+26922194450352 v^{40} \\
& -11404592789688 v^{44}+3756782036502 v^{48}-421486536163500 v^{28} \\
& -1062657813600 v^{52}-45397293396 v^{60}+290922851196 v^{56}-1976417606251692 v^{20} \\
& +2525883800478795 v^{16}-2045958218223822 v^{12}-68193367053291 v^{4} \\
& +518822412814782 v^{8}-1129718145924+703601320689252 v^{24}+20 v^{84}-2958074 v^{76} \\
& -1437073423 v^{68}+13224991788 v^{64}+175580978 v^{72}+140423 v^{80}
\end{aligned}
$$

$$
\begin{aligned}
& \mathcal{Q}_{8}=8 v^{80}-412 v^{7} 6+1130201 v^{72}-36420582 v^{68}+661666116 v^{64}-4050893934 v^{60} \\
& +31716802512 v^{56}-92949815898 v^{52}+550493727984 v^{48}-1678881463974 v^{44} \\
& +4547507588514 v^{40}-13314555136710 v^{36}+9923931144288 v^{32}-82854890580186 v^{28} \\
& +1492061941800 v^{24}-51956321216238 v^{20}-427117486358664 v^{16} \\
& +308048735088522 v^{12}-243317414920563 v^{8}+4198088418804 v^{4}+1938652126956 .
\end{aligned}
$$

In [15] an expression for $f_{2}^{\prime} \equiv \hat{c}_{2}^{\prime}+\frac{1}{2} \hat{b}_{2}^{\prime}$ was presented. It is straightforward to integrate it to obtain (imposing the proper boundary condition, i.e., $f_{2}(v) \rightarrow 0$ as 


$$
\begin{aligned}
& \left.v \rightarrow 0_{+}\right) \\
& f_{2}=\frac{\sqrt{3}\left(\delta_{1}-954\right)}{288} \operatorname{arctanh} \frac{v^{2}}{\sqrt{3}}+\frac{3475 \sqrt{3}\left(v^{4}-3\right)}{128\left(3+v^{4}\right)} \arctan \frac{v^{2}}{\sqrt{3}} \\
& -\frac{\hat{\eta}_{0}\left(1080 \hat{\eta}_{0}+\delta_{2}-3 \hat{\eta}_{0} \delta_{1}\right)}{24} \ln \frac{3-v^{4}}{3+v^{4}}+\frac{v^{2}}{1152\left(3+v^{4}\right)^{4}\left(v^{8}-9\right)^{4}}\left(-2125764 \delta_{1}\right. \\
& +\left(1417176 \delta_{1} C_{0}+272097792 \hat{\eta}_{0}^{2} \delta_{1}+226521411840 \hat{\eta}_{0}^{2}-51018336 \hat{\eta}_{0} \delta_{2}+408146688 C_{0}\right. \\
& \left.+17006112 \delta_{3}\right) v^{2}+\left(1180980 \delta_{1} C_{0}+272097792 C_{0}+158723712 \hat{\eta}_{0}^{2} \delta_{1}-39680928 \hat{\eta}_{0} \delta_{2}\right. \\
& \left.+17006112 \delta_{3}+174686782464 \hat{\eta}_{0}^{2}\right) v^{6}+\left(11074698999-4960116 \delta_{1}\right) v^{4}+(-8468512335 \\
& \left.-2598156 \delta_{1}\right) v^{8}+\left(-314928 \delta_{1} C_{0}-100147104 \hat{\eta}_{0}^{2} \delta_{1}-2857026816 C_{0}+13226976 \hat{\eta}_{0} \delta_{2}\right. \\
& \left.-816701522688 \hat{\eta}_{0}^{2}-1889568 \delta_{3}\right) v^{10}+\left(3564 \delta_{1}+91909647\right) v^{32}+\left(-165888 \hat{\eta}_{0}^{2} \delta_{1}\right. \\
& \left.-597756672 \hat{\eta}_{0}^{2}+3732480 C_{0}-1512 \delta_{1} C_{0}+44064 \hat{\eta}_{0} \delta_{2}-28512 \delta_{3}\right) v^{34}+\left(866052 \delta_{1}\right. \\
& +22334044221) v^{12}+\left(-74952864 \hat{\eta}_{0}^{2} \delta_{1}-498636 \delta_{1} C_{0}+3355872768 C_{0}+17006112 \hat{\eta}_{0} \delta_{2}\right. \\
& \left.+1203035037696 \hat{\eta}_{0}^{2}-6928416 \delta_{3}\right) v^{14}+\left(6110429886+997272 \delta_{1}\right) v^{16}+\left(419904 \hat{\eta}_{0} \delta_{2}\right. \\
& \left.+8398080 \hat{\eta}_{0}^{2} \delta_{1}-1375605504 C_{0}-1259712 \delta_{3}-34992 \delta_{1} C_{0}-1340988618240 \hat{\eta}_{0}^{2}\right) v^{18} \\
& +\left(979776 \delta_{3}+481310760960 \hat{\eta}_{0}^{2}+75816 \delta_{1} C_{0}+10077696 C_{0}-2659392 \hat{\eta}_{0} \delta_{2}\right. \\
& \left.+12877056 \hat{\eta}_{0}^{2} \delta_{1}\right) v^{22}+\left(87480 \delta_{1}+9442000710\right) v^{20}+\left(6220800 \hat{\eta}_{0}^{2}-2592 \hat{\eta}_{0} \delta_{2}\right. \\
& \left.+17280 \hat{\eta}_{0}^{2} \delta_{1}+36 \delta_{1} C_{0}-864 \delta_{3}\right) v^{38}+\left(-3125709+1404 \delta_{1}\right) v^{36}+\left(-1440 \hat{\eta}_{0} \delta_{2}+864 \delta_{3}\right. \\
& \left.+6324480 \hat{\eta}_{0}^{2}+48 \delta_{1} C_{0}+6624 \hat{\eta}_{0}^{2} \delta_{1}\right) v^{42}+\left(36 \delta_{1}+645597\right) v^{40}+\left(-12 \delta_{1}+105273\right) v^{44} \\
& +\left(480 \hat{\eta}_{0}^{2} \delta_{1}+96 \delta_{3}+4 \delta_{1} C_{0}-96 \hat{\eta}_{0} \delta_{2}+552960 \hat{\eta}_{0}^{2}\right) v^{46}+\left(-110808 \delta_{1}+3107022786\right) v^{24} \\
& +\left(-121224605184 \hat{\eta}_{0}^{2}-419904 \hat{\eta}_{0} \delta_{2}+153964800 C_{0}+15552 \delta_{1} C_{0}+886464 \hat{\eta}_{0}^{2} \delta_{1}\right. \\
& \left.+326592 \delta_{3}\right) v^{26}+\left(-25272 \delta_{1}+208396314\right) v^{28}+\left(171072 \hat{\eta}_{0} \delta_{2}-917568 \hat{\eta}_{0}^{2} \delta_{1}\right. \\
& \left.\left.-42923520 C_{0}+7936745472 \hat{\eta}_{0}^{2}-4536 \delta_{1} C_{0}-46656 \delta_{3}\right) v^{30}+18648796131\right) \text {. }
\end{aligned}
$$


We can further use a constraint at the second order (similar to (3.6)) to find

$$
\begin{aligned}
& \hat{a}_{2}^{\prime}=-\frac{10425 v^{3}\left(v^{8}+2 v^{4}+9\right) \sqrt{3}}{8\left(v^{8}-9\right)^{2}} \arctan \frac{v^{2}}{\sqrt{3}}+\frac{v^{3}}{72\left(v^{8}-9\right)^{5}\left(3+v^{4}\right)^{4}}\left(76527504 \delta_{3}\right. \\
& +1836660096 C_{0}+1101996057600 \hat{\eta}_{0}^{2}+994857552 \hat{\eta}_{0}^{2} \delta_{1}-153055008 \hat{\eta}_{0} \delta_{2} \\
& +6377292 \delta_{1} C_{0}+\left(-864 \hat{\eta}_{0} \delta_{2}+12 \delta_{1} C_{0}+576 \hat{\eta}_{0}^{2} \delta_{1}-2016 \delta_{3}\right) v^{44}+\left(119042784 \delta_{3}\right. \\
& -289103904 \hat{\eta}_{0} \delta_{2}+1851353376768 \hat{\eta}_{0}^{2}+1938696768 \hat{\eta}_{0}^{2} \delta_{1}+3265173504 C_{0} \\
& \left.+10628820 \delta_{1} C_{0}\right) v^{4}+\left(-339560128512 \hat{\eta}_{0}^{2}-3129840 \hat{\eta}_{0}^{2} \delta_{1}+674179200 C_{0}\right. \\
& \left.+15228 \delta_{1} C_{0}+190512 \delta_{3}-738720 \hat{\eta}_{0} \delta_{2}\right) v^{32}+\left(1912896 \hat{\eta}_{0} \delta_{2}+2034579326976 \hat{\eta}_{0}^{2}\right. \\
& \left.+606528 \delta_{3}-21384 \delta_{1} C_{0}-22581504 \hat{\eta}_{0}^{2} \delta_{1}-2058089472 C_{0}\right) v^{28}+(96435034542 \\
& \left.+1749600 \delta_{1}\right) v^{22}+\left(194755671-17712 \delta_{1}\right) v^{38}-\left(24091992 \delta_{1}-445385644605\right) v^{10} \\
& +\left(24383227336704 \hat{\eta}_{0}^{2}+69838433280 C_{0}-5668704 \delta_{3}+2913084 \delta_{1} C_{0}\right. \\
& \left.-179508960 \hat{\eta}_{0} \delta_{2}+1795089600 \hat{\eta}_{0}^{2} \delta_{1}\right) v^{12}+\left(-25509168 \delta_{1}-86716822293\right) v^{6} \\
& -144 \delta_{3} v^{48}+\left(18828186993-12282192 \delta_{1}\right) v^{14}+\left(-9977140749312 \hat{\eta}_{0}^{2}\right. \\
& \left.-78941952 C_{0}-71313696 \hat{\eta}_{0}^{2} \delta_{1}+16096320 \hat{\eta}_{0} \delta_{2}-274104 \delta_{1} C_{0}\right) v^{24}+\left(5598720 C_{0}\right. \\
& \left.-8640 \delta_{3}+468 \delta_{1} C_{0}-4752 \hat{\eta}_{0}^{2} \delta_{1}-28512 \hat{\eta}_{0} \delta_{2}-618098688 \hat{\eta}_{0}^{2}\right) v^{40}+(314464150422 \\
& \left.-1679616 \delta_{1}\right) v^{18}+\left(-27864 \delta_{1}+41837067\right) v^{3} 4-\left(57209092209+12754584 \delta_{1}\right) v^{2} \\
& +\left(178848 \delta_{1}+11908840482\right) v^{30}+\left(21413128771584 \hat{\eta}_{0}^{2}-3359232 \hat{\eta}_{0}^{2} \delta_{1}-5458752 \delta_{3}\right. \\
& \left.+18280940544 C_{0}-647352 \delta_{1} C_{0}+28133568 \hat{\eta}_{0} \delta_{2}\right) v^{20}+\left(7776 \delta_{3}-212544 \hat{\eta}_{0}^{2} \delta_{1}\right. \\
& \left.-261792 \hat{\eta}_{0} \delta_{2}-93312000 C_{0}+16858865664 \hat{\eta}_{0}^{2}+4644 \delta_{1} C_{0}\right) v^{36}+\left(1038096 \delta_{1}\right. \\
& +32981460282) v^{26}+\left(7794468 \delta_{1} C_{0}-33604077312 C_{0}+2406364848 \hat{\eta}_{0}^{2} \delta_{1}\right. \\
& \left.-8771888618496 \hat{\eta}_{0}^{2}-300441312 \hat{\eta}_{0} \delta_{2}+56687040 \delta_{3}\right) v^{8}+\left(-336 \delta_{1}-357507\right) v^{46} \\
& +\left(-16 \delta_{1}-4608\right) v^{50}+\left(-28973376 \hat{\eta}_{0} \delta_{2}+619148448 \hat{\eta}_{0}^{2} \delta_{1}-54268392960 C_{0}\right. \\
& \left.\left.-15431472 \delta_{3}-36532030952448 \hat{\eta}_{0}^{2}-52488 \delta_{1} C_{0}\right) v^{16}+\left(-4094631-3384 \delta_{1}\right) v^{42}\right) \text {. }
\end{aligned}
$$

Using (3.2)-(3.6) we can obtain a decoupled equation for $\hat{a}_{3}$ :

$$
0=\hat{a}_{3}^{\prime \prime}+\frac{5 v^{16}+18 v^{12}+216 v^{8}+126 v^{4}+243}{\left(v^{8}-9\right) v\left(v^{4}+2 v^{2}+3\right)\left(v^{4}-2 v^{2}+3\right)} \hat{a}_{3}^{\prime}+\mathcal{J}_{\hat{a}_{3}},
$$




$$
\begin{aligned}
\mathcal{J}_{\hat{a}_{3}}= & -\frac{768\left(v^{8}+18 v^{4}+9\right) v^{6} \hat{\eta}_{0}}{\left(v^{8}-9\right)^{2}\left(v^{8}+2 v^{4}+9\right)} f_{2}^{\prime}-\frac{4 v^{3} \mathcal{R}_{1}}{3\left(v^{4}-2 v^{2}+3\right)^{2}\left(v^{4}+2 v^{2}+3\right)^{2}\left(v^{8}-9\right)^{6}\left(3+v^{4}\right)} \\
& \times a_{3}^{\prime}-\frac{16 v^{6} \sqrt{3} \mathcal{R}_{2}}{9\left(v^{8}-9\right)^{6}\left(3+v^{4}\right)^{2}\left(v^{4}-2 v^{2}+3\right)^{2}\left(v^{4}+2 v^{2}+3\right)^{2}} \operatorname{arctanh} \frac{v^{2}}{\sqrt{3}} \\
& -\frac{125100 v^{6} \hat{\eta}_{0} \sqrt{3} \mathcal{R}_{3}}{\left(v^{8}-9\right)^{4}\left(v^{4}-3\right)^{2}\left(v^{4}-2 v^{2}+3\right)\left(v^{4}+2 v^{2}+3\right)} \arctan \frac{v^{2}}{\sqrt{3}} \\
& +\frac{\mathcal{R}_{4}}{27\left(v^{8}-9\right)^{5}\left(v^{4}-3\right)\left(v^{4}-2 v^{2}+3\right)^{2}\left(v^{4}+2 v^{2}+3\right)^{2}} \ln \frac{3-v^{4}}{3+v^{4}} \\
& +\frac{2 \hat{\eta}_{0} \delta_{1} v^{4} \mathcal{R}_{5}}{9\left(v^{8}-9\right)^{6}\left(v^{4}-2 v^{2}+3\right)^{2}\left(v^{4}+2 v^{2}+3\right)^{2}} \\
& +\frac{192 v^{6} \hat{\eta}_{0} \delta_{3} \mathcal{R}_{7}}{27\left(v^{8}-9\right)^{5}\left(v^{4}-3\right)\left(v^{4}-2 v^{2}+3\right)\left(v^{4}+2 v^{2}+3\right)} \\
& -\frac{4 v^{4} \hat{\eta}_{0} \mathcal{R}_{8}}{\left(v^{8}-9\right)^{4}\left(v^{4}-3\right)^{2}\left(v^{4}-2 v^{2}+3\right)\left(v^{4}+2 v^{2}+3\right)} \\
& +\frac{\left.v^{8}-9\right)^{7}\left(v^{4}+2 v^{2}+3\right)^{2}\left(v^{4}-2 v^{2}+3\right)^{2}\left(3+v^{4}\right)^{4}}{\left(v^{4}\right.}
\end{aligned}
$$

with

$$
\begin{aligned}
& \mathcal{R}_{1}=14348907 \delta_{1}+8264970432+\left(-1435349865024+90876411 \delta_{1}\right) v^{4}+\left(46235367 \delta_{1}\right. \\
& +6473308508352) v^{8}+\left(-23206257 \delta_{1}-11722074916032\right) v^{12}+(11877204669696 \\
& \left.-16592769 \delta_{1}\right) v^{16}+\left(1043199 \delta_{1}-7723609200000\right) v^{20}+\left(1646811 \delta_{1}\right. \\
& +3567784040064) v^{24}+\left(-85293 \delta_{1}-1321470640512\right) v^{28}+\left(-28431 \delta_{1}\right. \\
& +440473837248) v^{32}+\left(-132105017664+60993 \delta_{1}\right) v^{36}+\left(4293 \delta_{1}+31786872768\right) v^{40} \\
& +\left(-5435190720-7587 \delta_{1}\right) v^{44}+\left(594864000-1179 \delta_{1}\right) v^{48}+\left(261 \delta_{1}-36391680\right) v^{52} \\
& +\left(933120+57 \delta_{1}\right) v^{56}+v^{60} \delta_{1}
\end{aligned}
$$




$$
\begin{aligned}
& \mathcal{R}_{2}=-16529940864 \hat{\eta}_{0}+4782969 \delta_{2}-43046721 \hat{\eta}_{0} \delta_{1}+\left(13817466 \delta_{2}+532631427840 \hat{\eta}_{0}\right. \\
& \left.-149866362 \hat{\eta}_{0} \delta_{1}\right) v^{4}+\left(8680203 \delta_{2}+3526999604352 \hat{\eta}_{0}-101505231 \hat{\eta}_{0} \delta_{1}\right) v^{8} \\
& +\left(6608711172096 \hat{\eta}_{0}-1653372 \delta_{2}+7794468 \hat{\eta}_{0} \delta_{1}\right) v^{12}+\left(-3247695 \delta_{2}+26394903 \hat{\eta}_{0} \delta_{1}\right. \\
& \left.-1430009945856 \hat{\eta}_{0}\right) v^{16}+\left(5786802 \hat{\eta}_{0} \delta_{1}+5096210245632 \hat{\eta}_{0}-800442 \delta_{2}\right) v^{20} \\
& -\left(1358127 \hat{\eta}_{0} \delta_{1}+277988205312 \hat{\eta}_{0}-247131 \delta_{2}\right) v^{24}+\left(726514541568 \hat{\eta}_{0}+180792 \delta_{2}\right. \\
& \left.-542376 \hat{\eta}_{0} \delta_{1}\right) v^{28}+\left(-30848107392 \hat{\eta}_{0}+27459 \delta_{2}-13851 \hat{\eta}_{0} \delta_{1}\right) v^{32}+\left(-9882 \delta_{2}\right. \\
& \left.+62892101376 \hat{\eta}_{0}-12150 \hat{\eta}_{0} \delta_{1}\right) v^{36}+\left(-1974761856 \hat{\eta}_{0}-4455 \delta_{2}-9477 \hat{\eta}_{0} \delta_{1}\right) v^{40} \\
& +\left(-252 \delta_{2}+324 \hat{\eta}_{0} \delta_{1}+1007023104 \hat{\eta}_{0}\right) v^{44}+\left(147 \delta_{2}+837 \hat{\eta}_{0} \delta_{1}+60466176 \hat{\eta}_{0}\right) v^{48} \\
& +\left(1119744 \hat{\eta}_{0}+126 \hat{\eta}_{0} \delta_{1}+26 \delta_{2}\right) v^{52}+\left(3 \hat{\eta}_{0} \delta_{1}+\delta_{2}\right) v^{56}, \\
& \mathcal{R}_{3}=7 v^{28}-3 v^{24}-93 v^{20}-63 v^{16}+1269 v^{12}-4617 v^{8}+6561 v^{4}+2187, \\
& \mathcal{R}_{4}=-51656065200 \hat{\eta}_{0}-23914845 \delta_{2}+\left(459165024 \hat{\eta}_{0}^{3} \delta_{1}-153055008 \hat{\eta}_{0}^{2} \delta_{2}\right. \\
& \left.-165299408640 \hat{\eta}_{0}^{3}\right) v^{6}+71744535 \hat{\eta}_{0} \delta_{1}+\left(596465856 \hat{\eta}_{0}+684 \delta_{2}-2970 \hat{\eta}_{0} \delta_{1}\right) v^{44} \\
& +\left(-12397455648 \hat{\eta}_{0}+100442349 \hat{\eta}_{0} \delta_{1}-18068994 \delta_{2}\right) v^{4}+\left(1948617 \delta_{2}\right. \\
& \left.-3275759808720 \hat{\eta}_{0}+39326634 \hat{\eta}_{0} \delta_{1}\right) v^{8}+\left(-312232216320 \hat{\eta}_{0}^{3}+867311712 \hat{\eta}_{0}^{3} \delta_{1}\right. \\
& \left.-289103904 \hat{\eta}_{0}^{2} \delta_{2}\right) v^{10}+\left(-87 \hat{\eta}_{0} \delta_{1}-102816 \hat{\eta}_{0}-34 \delta_{2}\right) v^{52}+\left(6022998 \hat{\eta}_{0} \delta_{1}+4487724 \delta_{2}\right. \\
& \left.+3920759121600 \hat{\eta}_{0}\right) v^{12}+\left(1539 \delta_{2}-3078 \hat{\eta}_{0} \delta_{1}-13050184752 \hat{\eta}_{0}\right) v^{40}+\left(28512 \hat{\eta}_{0}^{2} \delta_{2}\right. \\
& \left.-85536 \hat{\eta}_{0}^{3} \delta_{1}+30792960 \hat{\eta}_{0}^{3}\right) v^{42}+\left(1259712 \hat{\eta}_{0}^{2} \delta_{2}+1360488960 \hat{\eta}_{0}^{3}-3779136 \hat{\eta}_{0}^{3} \delta_{1}\right) v^{22} \\
& +\left(67344203520 \hat{\eta}_{0}^{3}-187067232 \hat{\eta}_{0}^{3} \delta_{1}+62355744 \hat{\eta}_{0}^{2} \delta_{2}\right) v^{14}+\left(368145 \hat{\eta}_{0} \delta_{1}-12879 \delta_{2}\right. \\
& \left.-282820705488 \hat{\eta}_{0}\right) v^{32}+\left(411505920 \hat{\eta}_{0}^{3}+381024 \hat{\eta}_{0}^{2} \delta_{2}-1143072 \hat{\eta}_{0}^{3} \delta_{1}\right) v^{38}-\left(115911 \delta_{2}\right. \\
& \left.+2547194665968 \hat{\eta}_{0}+2965572 \hat{\eta}_{0} \delta_{1}\right) v^{24}+\left(2592 \hat{\eta}_{0}^{3} \delta_{1}-933120 \hat{\eta}_{0}^{3}-864 \hat{\eta}_{0}^{2} \delta_{2}\right) v^{50}-\left(5 \delta_{2}\right. \\
& \left.+15120 \hat{\eta}_{0}\right) v^{56}+\left(-64152 \delta_{2}+96228 \hat{\eta}_{0} \delta_{1}+143399735424 \hat{\eta}_{0}\right) v^{28}+\left(50388480 \hat{\eta}_{0}^{3}\right. \\
& \left.+46656 \hat{\eta}_{0}^{2} \delta_{2}-139968 \hat{\eta}_{0}^{3} \delta_{1}\right) v^{34}+\left(-765 \hat{\eta}_{0} \delta_{1}+33 \delta_{2}-55887408 \hat{\eta}_{0}\right) v^{48}+\left(44064 \hat{\eta}_{0}^{3} \delta_{1}\right. \\
& \left.-15863040 \hat{\eta}_{0}^{3}-14688 \hat{\eta}_{0}^{2} \delta_{2}\right) v^{46}+\left(26453952 \hat{\eta}_{0}^{3} \delta_{1}-9523422720 \hat{\eta}_{0}^{3}-8817984 \hat{\eta}_{0}^{2} \delta_{2}\right) v^{26} \\
& +\left(92588832 \hat{\eta}_{0}^{2} \delta_{2}-277766496 \hat{\eta}_{0}^{3} \delta_{1}+99995938560 \hat{\eta}_{0}^{3}\right) v^{18}+\left(68283 \hat{\eta}_{0} \delta_{1}+162 \delta_{2}\right. \\
& \left.+23803727904 \hat{\eta}_{0}\right) v^{36}+\left(1924904811168 \hat{\eta}_{0}-5570289 \hat{\eta}_{0} \delta_{1}+13122 \delta_{2}\right) v^{20} \\
& +\left(8817984 \hat{\eta}_{0}^{3} \delta_{1}-3174474240 \hat{\eta}_{0}^{3}-2939328 \hat{\eta}_{0}^{2} \delta_{2}\right) v^{30}+\left(-1121931 \hat{\eta}_{0} \delta_{1}\right. \\
& \left.-9513261568080 \hat{\eta}_{0}+1121931 \delta_{2}\right) v^{16} \text {, }
\end{aligned}
$$




$$
\begin{aligned}
& \mathcal{R}_{5}=-\left(7164612 C_{0}+4472292528 \hat{\eta}_{0}^{2}\right) v^{18}+119062467 v^{16}+223087122 v^{12} \\
&+ 315675954 v^{8}+74933181 v^{4}-9198 v^{44}-1583469 v^{32}-82638 v^{40}-430029 v^{36} \\
&- 915 v^{48}+13 v^{52}-2032452 v^{28}+34031907 v^{20}+4452732 v^{24}+\left(115560 C_{0}\right. \\
&-\left.16708032 \hat{\eta}_{0}^{2}\right) v^{34}+\left(-46530612 C_{0}-10489936752 \hat{\eta}_{0}^{2}\right) v^{10}+\left(17712 C_{0}-3045600 \hat{\eta}_{0}^{2}\right) v^{38} \\
&+\left(52704 \hat{\eta}_{0}^{2}+648 C_{0}\right) v^{46}+\left(12 C_{0}+1872 \hat{\eta}_{0}^{2}\right) v^{50}+\left(-118646208 \hat{\eta}_{0}^{2}+470448 C_{0}\right) v^{30} \\
&+\left(110160 \hat{\eta}_{0}^{2}+2268 C_{0}\right) v^{42}+\left(-10507887648 \hat{\eta}_{0}^{2}-15903864 C_{0}\right) v^{14}+\left(-482772960 \hat{\eta}_{0}^{2}\right. \\
&+\left.173016 C_{0}\right) v^{26}-\left(25509168 C_{0}+3520265184 \hat{\eta}_{0}^{2}\right) v^{2}-\left(2133532224 \hat{\eta}_{0}^{2}+2799360 C_{0}\right) v^{22} \\
&-\left(127545840 C_{0}+21070572768 \hat{\eta}_{0}^{2}\right) v^{6}+23914845, \\
& \mathcal{R}_{6}=-885735+\left(236196 C_{0}+62355744 \hat{\eta}_{0}^{2}\right) v^{2}-885735 v^{4}+\left(866052 C_{0}\right. \\
&\left.+288159120 \hat{\eta}_{0}^{2}\right) v^{6}-2302911 v^{8}+\left(-26453952 \hat{\eta}_{0}^{2}-157464 C_{0}\right) v^{10}-177147 v^{12} \\
&+\left(66624768 \hat{\eta}_{0}^{2}-134136 C_{0}\right) v^{14}+275562 v^{16}+\left(-5412096 \hat{\eta}_{0}^{2}+27216 C_{0}\right) v^{18} \\
&+92826 v^{20}+\left(9072 C_{0}-1594080 \hat{\eta}_{0}^{2}\right) v^{22}+7938 v^{24}-\left(4968 C_{0}+1684800 \hat{\eta}_{0}^{2}\right) v^{26} \\
&-2214 v^{28}+\left(-31104 \hat{\eta}_{0}^{2}-648 C_{0}\right) v^{30}-963 v^{32}+\left(-50976 \hat{\eta}_{0}^{2}+396 C_{0}\right) v^{34}-531 v^{36} \\
&+\left(-1584 \hat{\eta}_{0}^{2}+12 C_{0}\right) v^{38}-3 v^{40}+v^{44}, \\
& \mathcal{R}_{7}=7 v^{28}-3 v^{24}-93 v^{20}-63 v^{16}+1269 v^{12}-4617 v^{8}+6561 v^{4}+2187, \\
&(\mathrm{~B} .19) \\
&
\end{aligned}
$$




$$
\begin{aligned}
& \mathcal{R}_{8}=1750802750915778 v^{16}-547909426568250 v^{12}+551849751221706 v^{8} \\
& -3229924616793 v^{4}+10220357329866 v^{44}+252995054673834 v^{32} \\
& +32427971352054 v^{40}+94323062131416 v^{36}+1924492298742 v^{48}+491409014598 v^{52} \\
& +392340630598470 v^{28}+310374023297562 v^{20}+903540325216554 v^{24} \\
& +31603772430 v^{56}+11854881954 v^{60}-177976890 v^{64}+55953441 v^{68}-376 v^{76} \\
& +185736 v^{72}+16 v^{80}+\left(-77182514538916608 \hat{\eta}_{0}^{2}-361083701553408 C_{0}\right) v^{14} \\
& +\left(6169681384704 C_{0}+20228383300939776 \hat{\eta}_{0}^{2}\right) v^{26}+\left(-39822513408 \hat{\eta}_{0}^{2}\right. \\
& \left.+685117440 C_{0}\right) v^{62}+\left(117792358596864 C_{0}+19949787269471232 \hat{\eta}_{0}^{2}\right) v^{10} \\
& +\left(211985675693568 C_{0}+64589051749911552 \hat{\eta}_{0}^{2}\right) v^{18}-\left(23950080 C_{0}+403273728 \hat{\eta}_{0}^{2}\right) \\
& \times v^{66}-\left(4040529154973568 \hat{\eta}_{0}^{2}+29968782786432 C_{0}\right) v^{6}+\left(8159616 \hat{\eta}_{0}^{2}+746496 C_{0}\right) v^{70} \\
& +\left(24405013822464 \hat{\eta}_{0}^{2}-7899545088 C_{0}\right) v^{50}+\left(107114016798720 \hat{\eta}_{0}^{2}\right. \\
& \left.+297538935552 C_{0}\right) v^{2}+\left(-281691198720 C_{0}+348792330451968 \hat{\eta}_{0}^{2}\right) v^{42} \\
& +\left(20993178240 C_{0}-6791658865920 \hat{\eta}_{0}^{2}\right) v^{54}+\left(-3560308992 C_{0}+487741077504 \hat{\eta}_{0}^{2}\right) v^{58} \\
& +\left(-7095537792 C_{0}-2444692311194112 \hat{\eta}_{0}^{2}\right) v^{38}+\left(2463432321024 C_{0}\right. \\
& \left.+3589000230500352 \hat{\eta}_{0}^{2}\right) v^{34}+\left(-15313028838912 C_{0}-16874613168627456 \hat{\eta}_{0}^{2}\right) v^{30} \\
& +\left(-177353046892800 \hat{\eta}_{0}^{2}+190336884480 C_{0}\right) v^{46}-\left(138461655146112 C_{0}\right. \\
& \left.+66703936055747328 \hat{\eta}_{0}^{2}\right) v^{22}+697356880200 \\
& +
\end{aligned}
$$

For the computation of $C$ we actually need only the asymptotic solution of $\hat{a}_{3}^{\prime}(v)$ as $x \equiv 3^{1 / 4}-v \rightarrow 0_{+}$. Using (B.11)-(B.20) we find:

$$
\begin{aligned}
& \left.\frac{d \hat{a}_{3}(v)}{d v}\right|_{x \equiv 3^{1 / 4}-v \rightarrow 0_{+}}=\frac{40 \hat{\eta}_{0}\left(-18 \hat{\eta}_{0}^{2}+\sqrt{3}\right)}{3^{3 / 4}} \frac{1}{x^{6}}-\frac{\hat{\eta}_{0}}{48}\left(256 C_{0}-512 \sqrt{3} \ln 2+1760 \sqrt{3}\right. \\
& \left.-\left(6432+3 \delta_{1}\right) \hat{\eta}_{0}^{2}\right) \frac{1}{x^{5}}+\frac{3^{1 / 4} \hat{\eta}_{0}}{1152}\left(-18432 \ln 2-12096-12 \delta_{1}+\sqrt{3} C_{0}\left(3360+\delta_{1}\right)\right. \\
& \left.-24 \hat{\eta}_{0} \sqrt{3} \delta_{2}+24 \sqrt{3}\left(15792+5 \delta_{1}\right) \hat{\eta}_{0}^{2}\right) \frac{1}{x^{4}}+\left(\frac{1}{12} 3^{1 / 4}\left(288+\delta_{1}\right) \mathcal{A}_{3}+\frac{1}{5184} \sqrt{3}\left(38880 \hat{\eta}_{0}\right.\right. \\
& \left.-2 \delta_{2}+9 \hat{\eta}_{0} \delta_{1}\right) C_{0}+\frac{1}{96} \sqrt{3}\left(67872+13 \delta_{1}\right) \hat{\eta}_{0}^{3}-\frac{17}{432} \sqrt{3} \delta_{2} \hat{\eta}_{0}^{2}+\left(\frac{1025}{24}-46 \ln 2\right. \\
& \left.\left.+\frac{1}{36} \sqrt{3} \delta_{3}+\frac{3475}{256} \pi\right) \hat{\eta}_{0}+\frac{1}{216} \delta_{2}\right) \frac{1}{x^{3}}+\hat{\mathcal{A}}_{3} \frac{1}{x^{2}}+\mathcal{O}\left(x^{-1}\right)
\end{aligned}
$$

where $\hat{\mathcal{A}}_{3}$ must be fixed so to satisfy the boundary condition (3.1). 


\section{Source terms for the framework of Kinoshita et al}

Order - 0:

$$
\begin{aligned}
& S_{A}^{0}=180 y^{10}\left(16-23 y^{4}\right), \\
& S_{B}^{0}=-4320 y^{10}, \\
& S_{C}^{0}=0 .
\end{aligned}
$$

Order - 1:

$$
\begin{aligned}
S_{A}^{1}= & -\left(33 w\left(y^{3}+y^{2}+y+1\right)^{2}\right)^{-1} \\
& 4 y^{6}\left(11 A_{1}^{0}\left(y^{3}+2 y^{2}+3 y+4\right)-3 y^{4}\left(74945 y^{11}+191854 y^{10}+308763 y^{9}\right.\right. \\
& +425672 y^{8}+370056 y^{7}+209256 y^{6}+48456 y^{5}-112344 y^{4}-153504 y^{3} \\
& \left.\left.-109728 y^{2}-65952 y-22176\right)\right) \\
S_{B}^{1}= & \frac{8640 y^{10}(11+26 y)}{11 w} \\
S_{C}^{1}= & -\left(33 w(y-1)\left(y^{3}+y^{2}+y+1\right)^{3}\right)^{-1} \\
& \left(2 y ^ { 3 } \left(3 \left(84 y^{10}+168 y^{9}+252 y^{8}+2541 y^{7}-754 y^{6}-4049 y^{5}-7344 y^{4}\right.\right.\right. \\
& \left.\left.\left.-15624 y^{3}-12528 y^{2}-9432 y-6336\right) y^{7}+11 A_{1}^{0}\left(3 y^{2}+2 y+1\right)\right)\right) .
\end{aligned}
$$

\section{References}

[1] J. D. Bjorken, Phys. Rev. D 27, 140 (1983).

[2] I. Muller, Z. Phys. 198 (1967) 329.

[3] W. Israel and J. M. Stewart, Annals Phys. 118 (1979) 341.

[4] A. Muronga, Phys. Rev. C 69, 034903 (2004) [arXiv:nucl-th/0309055].

[5] J. M. Maldacena, Adv. Theor. Math. Phys. 2, 231 (1998) [Int. J. Theor. Phys. 38, 1113 (1999)] [arXiv:hep-th/9711200].

[6] O. Aharony, S. S. Gubser, J. M. Maldacena, H. Ooguri and Y. Oz, Phys. Rept. 323, 183 (2000) [arXiv:hep-th/9905111].

[7] D. T. Son and A. O. Starinets, Ann. Rev. Nucl. Part. Sci. 57, 95 (2007) [arXiv:0704.0240 [hep-th]]. 
[8] R. Baier, P. Romatschke, D. T. Son, A. O. Starinets and M. A. Stephanov, JHEP 0804, 100 (2008) [arXiv:0712.2451 [hep-th]].

[9] S. Bhattacharyya, V. E. Hubeny, S. Minwalla and M. Rangamani, JHEP 0802, 045 (2008) [arXiv:0712.2456 [hep-th]].

[10] A. Buchel, AIP Conf. Proc. 1031, 196 (2008) [arXiv:0803.3421 [hep-th]].

[11] G. Policastro, D. T. Son and A. O. Starinets, Phys. Rev. Lett. 87, 081601 (2001) [arXiv:hep-th/0104066].

[12] A. Buchel, J. T. Liu and A. O. Starinets, Nucl. Phys. B 707, 56 (2005) [arXiv:hepth/0406264].

[13] P. Benincasa and A. Buchel, JHEP 0601, 103 (2006) [arXiv:hep-th/0510041].

[14] A. Buchel, Nucl. Phys. B 803, 166 (2008) [arXiv:0805.2683 [hep-th]].

[15] A. Buchel, Nucl. Phys. B 802, 281 (2008) [arXiv:0801.4421 [hep-th]].

[16] R. C. Myers, M. F. Paulos and A. Sinha, "Quantum corrections to eta/s," arXiv:0806.2156 [hep-th].

[17] M. F. Paulos, "Higher derivative terms including the Ramond-Ramond five-form" arXiv:0804.0763 [hep-th].

[18] A. Buchel and M. Paulos, "Relaxation time of a CFT plasma at finite coupling," arXiv:0806.0788 [hep-th].

[19] A. Buchel, Phys. Lett. B 665, 298 (2008) [arXiv:0804.3161 [hep-th]].

[20] A. Buchel, R. C. Myers, M. F. Paulos and A. Sinha, "Universal holographic hydrodynamics at finite coupling," arXiv:0808.1837 [hep-th].

[21] R. A. Janik and R. Peschanski, Phys. Rev. D 73, 045013 (2006) [arXiv:hepth/0512162].

[22] S. Nakamura and S. J. Sin, "A holographic dual of hydrodynamics," JHEP 0609, 020 (2006) [arXiv:hep-th/0607123].

[23] R. A. Janik, "Viscous plasma evolution from gravity using AdS/CFT," Phys. Rev. Lett. 98, 022302 (2007) [arXiv:hep-th/0610144]. 
[24] M. P. Heller and R. A. Janik, Phys. Rev. D 76, 025027 (2007) [arXiv:hepth/0703243].

[25] The Maple $^{\odot}$ worksheet containing the source terms $\left\{\mathcal{S}_{(3,1)} \cdots \mathcal{S}_{(3,7)}\right\}$ is available at http://abuchel.apmaths.uwo.ca/ public/research/s31s37.mw

[26] P. Benincasa, A. Buchel, M. P. Heller and R. A. Janik, Phys. Rev. D 77, 046006 (2008) [arXiv:0712.2025 [hep-th]].

[27] M. P. Heller, P. Surowka, R. Loganayagam, M. Spalinski and S. E. Vazquez, "On a consistent AdS/CFT description of boost-invariant plasma," arXiv:0805.3774 [hep-th].

[28] S. Kinoshita, S. Mukohyama, S. Nakamura and K. y. Oda, "A Holographic Dual of Bjorken Flow," arXiv:0807.3797 [hep-th].

[29] A. Buchel, Nucl. Phys. B 750, 45 (2006) [arXiv:hep-th/0604167].

[30] S. S. Gubser, I. R. Klebanov and A. A. Tseytlin, Nucl. Phys. B 534, 202 (1998) [arXiv:hep-th/9805156]. 